\title{
Regularization dependence of the OTOC. Which Lyapunov spectrum is the physical one?
}

\author{
Aurelio Romero-Bermúdez, Koenraad Schalm and Vincenzo Scopelliti \\ Instituut-Lorentz, $\triangle I T P$, Universiteit Leiden, \\ P.O. Box 9506, 2300 RA Leiden, The Netherlands \\ E-mail: romero@lorentz.leidenuniv.nl, kschalm@lorentz.leidenuniv.nl, \\ scopelliti@lorentz.leidenuniv.nl
}

ABSTRACT: We study the contour dependence of the out-of-time-ordered correlation function (OTOC) both in weakly coupled field theory and in the Sachdev-Ye-Kitaev (SYK) model. We show that its value, including its Lyapunov spectrum, depends sensitively on the shape of the complex time contour in generic weakly coupled field theories. For gapless theories with no thermal mass, such as SYK, the Lyapunov spectrum turns out to be an exception; their Lyapunov spectra do not exhibit contour dependence, though the full OTOCs do. Our result puts into question which of the Lyapunov exponents computed from the exponential growth of the OTOC reflects the actual physical dynamics of the system. We argue that, in a weakly coupled $\Phi^{4}$ theory, a kinetic theory argument indicates that the symmetric configuration of the time contour, namely the one for which the bound on chaos has been proven, has a proper interpretation in terms of dynamical chaos. Finally, we point out that a relation between these OTOCs and a quantity which may be measured experimentally — the Loschmidt echo — also suggests a symmetric contour configuration, with the subtlety that the inverse periodicity in Euclidean time is half the physical temperature. In this interpretation the chaos bound reads $\lambda \leq \frac{2 \pi}{\beta}=\pi T_{\text {physical }}$.

KeYwords: Quantum Dissipative Systems, AdS-CFT Correspondence, Holography and condensed matter physics (AdS/CMT)

ARXIV EPRINT: 1903.09595 


\section{Contents}

1 Introduction 1

2 A two-parameter family of extended Schwinger-Keldysh contours 4

2.1 The $\alpha$-contour 6

$\begin{array}{lll}2.2 & \text { OTOCS and physical observables in SK formalism } & 7\end{array}$

3 Contour dependence of the Lyapunov spectrum in a matrix $\Phi^{4}$ theory at $\begin{array}{lr}\text { weak coupling } & \mathbf{9}\end{array}$

$\begin{array}{ll}3.1 & \text { The contour dependence regulates the IR } \\ \end{array}$

$\begin{array}{lll}3.2 & \text { Kinetic theory interpretation of the } \alpha \text {-deformed OTOC } & 15\end{array}$

4 Contour dependence of the Lyapunov exponent in the SYK model 17

4.1 Study of the OTOC in SYK in the strongly coupled limit: conformal limit $\begin{array}{ll}\text { analysis } & 19\end{array}$

4.2 Study of the OTOC in SYK in the limit of large interaction order 20

5 The Lyapunov spectrum and the Loschmidt echo 22

$\begin{array}{lll}5.1 & \text { Loschmidt echo } & 23\end{array}$

6 Conclusion 26

$\begin{array}{lr}\text { A Numerical calculation in matrix model } & 28\end{array}$

\section{Introduction}

It has long been known that chaos, understood as the exponential sensitivity of the dynamics to initial conditions, does not have an immediate equivalent in the quantum dynamics governed by the Schrödinger equation. In quantum systems one needs to define quantum chaos in a more indirect way. One way to do so, is to measure the correlation between an operator $W(t)$ and some earlier perturbation $V(0)$ and compare this with the correlation where the perturbation $V(0)$ is performed after operator $W(t)$ is inserted:

$$
\left\langle\psi_{\text {final }}|W(t) V(0)| \psi_{\text {initial }}\right\rangle-\left\langle\psi_{\text {final }}|V(0) W(t)| \psi_{\text {initial }}\right\rangle=\left\langle\psi_{\text {final }}|[W(t), V(0)]| \psi_{\text {initial }}\right\rangle \text {. }
$$

Choosing $W(t)=q(t)$ and $V(0)=p(0)$ this commutator formally equals $[W(t), V(0)]=$ $i \hbar \frac{\partial q(t)}{\partial q(0)}$ and in that sense the above measures the sensitivity to initial conditions. The commutator is evaluated between two wave-functions, however. For a generic $\left|\psi_{\text {initial }}\right\rangle$ and 
$\left|\psi_{\text {final }}\right\rangle$, this is a complex amplitude that also depends on the details of both. An obvious step is to sum over final states, which converts this to an expectation value

$$
\begin{aligned}
C\left(t ; \psi_{\text {initial }}\right) & =\sum_{\text {final }}\left\langle\psi_{\text {initial }}\left|[W(t), V(0)]^{\dagger}\right| \psi_{\text {final }}\right\rangle\left\langle\psi_{\text {final }}|[W(t), V(0)]| \psi_{\text {initial }}\right\rangle \\
& =\left\langle\psi_{\text {initial }}\left|[W(t), V(0)]^{\dagger}[W(t), V(0)]\right| \psi_{\text {initial }}\right\rangle .
\end{aligned}
$$

To also isolate the dynamics driven by $V(0)$ and $W(t)$ as much from the details of the initial state, one can average over a suitable ensemble. A physically natural choice is the thermal one

$$
\begin{aligned}
C(t ; \beta) & =\sum_{\text {initial }} e^{-\beta E\left[\psi_{\text {initial }}\right]}\left\langle\psi_{\text {initial }}\left|[W(t), V(0)]^{\dagger}[W(t), V(0)]\right| \psi_{\text {initial }}\right\rangle \\
& =\operatorname{Tr} \rho_{\beta}[W(t), V(0)]^{\dagger}[W(t), V(0)] .
\end{aligned}
$$

This commutator-squared $C(t ; \beta)$ or, equivalently, this out-of-time ordered correlation function (OTOC) has been of much interest as a diagnostic of chaotic behaviour in many-body systems [1-3]. Specifically, if this OTOC has a regime where it exhibits an exponential time dependence, $C(t) \sim e^{\lambda t}$, this behaviour has been proposed to be a signature of chaos, with $\lambda$ being the quantum Lyapunov exponent. ${ }^{1}$ Moreover, this quantum Lyapunov exponent has been conjectured to be bounded from above $\lambda \leq 2 \pi k_{B} T / \hbar[3]$.

In practice most computations do not compute $C(t)$ as defined above. Rather one "smears" the thermal distribution between the two commutators [3, 4]

$$
C(t ; \beta)_{\text {regulated }} \equiv \operatorname{Tr}\left(\rho^{\frac{1}{2}}[W(t), V]^{\dagger} \rho^{\frac{1}{2}}[W(t), V]\right) .
$$

Mathematically, this has the advantage of being manifestly Hermitian (see e.g. [4]). The physical intuition is that in a QFT this correlation function naively suffers from a shortdistance divergences caused by the insertion of two operators at the same time. As chaos is in principle a long-time characteristic, the claim is that the information about chaos, and in particular the Lyapunov exponents $\lambda$, do not depend on this regularization $[3,4]$.

We will show that this intuition is incorrect, as was also pointed out earlier in [5] for the specific case of $2 \mathrm{D}$ fermions with quenched disorder. By explicit computation we will show that in the two-parameter family of "regularized" OTOCs

$$
C(t ; \beta)_{(\alpha, \sigma)} \equiv-\operatorname{Tr}\left[\rho^{1-\alpha-\sigma}\left(W^{\dagger}(t) \rho^{\sigma} V^{\dagger}-V^{\dagger} \rho^{\sigma} W^{\dagger}(t)\right) \rho^{\alpha-\sigma}\left(W(t) \rho^{\sigma} V-V \rho^{\sigma} W(t)\right)\right],
$$

the Lyapunov exponents are independent of $\sigma$ but do depend on $\alpha$. Our computation shows that this regularization dependence is an IR-effect and has nothing to do with shortdistance singularities. The more appropriate comparison for the regularization dependence of the OTOC is the proof in Schwinger-Keldysh theory that physical correlation functions are independent on the choice of contour. In Schwinger-Keldysh theory, there is a diagrammatic proof that physical Green's functions involving operator insertions either on

\footnotetext{
${ }^{1}$ Note that the Lyupanov exponent defined this way is in fact twice the chaos exponent one would surmise from the choice $W(t)=q(t), V(0)=p(0)$ with $q(t) \sim e^{\lambda_{\text {chaos }} t} q(0)$, i.e. $\lambda=2 \lambda_{\text {chaos }}$.
} 
only forward or only backward branches are independent of the contour due to energy conservation; this can be found in e.g. [6, 7]. The OTOC, however, is a correlation on a doubled Schwinger-Keldysh contour [8] and the two-body Green's functions involved in the commutator-squared involve operators inserted on both forward and backward branches. The arguments of $[6,7]$ do not generalize to prove that the correlation functions that appear in $C(t ; \beta)_{(\alpha, \sigma)}$ must be independent on the contour. Our explicit computation in section 3 shows that they indeed are not.

Gapless theories are notoriously more IR sensitive than gapped theories. Perhaps somewhat counterintuitively, our results show that weakly coupled gapless theories are in fact less contour-dependent than explicitly gapped theories, although the thermally generated mass does imbue a suppressed dependence. The SYK model on the other hand, which has been at the forefront of many OTOC studies, has no thermally induced mass. In this model specifically the contour dependence is extremely weak. In fact its Lyapunov spectrum turns out to be always contour-independent, as we show in section 4 .

Let us stress that the found contour dependence in generic models is not a pedantic point. As also pointed out by [5], OTOCs are now being measured either in numerical or actual physical experiments. Often one massages the regulator to be the most convenient for the set-up. For instance, Das et al. [9] use the canonical thermal OTOC $C(t ; \beta)_{(0,0)}$ in a numerical study, whereas a cold atom experiment measures a Loschmidt echo [10], which can be related to $C(t ; \beta)_{\left(\frac{1}{2}, 0\right)}$. As the theoretical prediction for these two correlation functions is different due to the regulator dependence, these two experimental results cannot be compared to each other.

Given the regularization dependence that we and [5] observe, the immediate question arises: which is the proper regularization that measures quantum chaos. As the previous paragraph shows, to some extent this is in the eye of the beholder. One can devise experimental set-ups that measure either. Nevertheless, we will argue that the OTOC that most closely reflects physical microscopic chaos is the symmetrized one $C(t ; \beta)_{\left(\frac{1}{2}, 0\right)}$ used originally for hermiticity reasons. Our argument rests on the following fact: in weakly coupled field theories the computation of any of the OTOCs $C(t ; \beta)_{(\alpha, \sigma)}$ can be cast in the form of a kinetic equation [11]. This kinetic equation reveals most closely the physical process one is actually computing. In terms of the kinetic equation, only the symmetrized OTOC with $\alpha=1 / 2$ can be understood as a microscopic unbiased "collision"-counter. Such unbiased collision counters have long been successfully proposed as tracking microscopic classical chaos [12, 13]. This is explained in section 3.2.

We conclude by showing the symmetric OTOC $C(t ; \beta)_{\left(\frac{1}{2}, 0\right)}$ regulated this way has a natural interpretation as a Loschmidt echo, rather than an expectation value in a thermal ensemble as in the introductory thought experiment. This has as subtle physical consequence that the physical temperature is set by twice the inverse periodicity in Euclidean time. In this interpretation the MSS bound reads

$$
\lambda \leq \frac{k_{B}}{\hbar} \frac{2 \pi}{\beta}=\frac{\pi k_{B} T_{\text {physical }}}{\hbar} .
$$




\section{A two-parameter family of extended Schwinger-Keldysh contours}

We will assume that $W(t)$ and $V(0)$ are hermitian from here on.

We formally consider the following regularization of the commutator-squared of eq. (1.3):

$$
\begin{aligned}
C(t ; \beta)_{(\alpha, \sigma)} & =\operatorname{Tr}\left[\mathcal{A}^{\dagger} \mathcal{A}\right] \geq 0, \\
\mathcal{A} & \equiv \rho^{\frac{\alpha-\sigma}{2}}[W(t), V(0)]_{\sigma} \rho^{\frac{1-\alpha-\sigma}{2}}, \quad[A, B]_{\sigma} \equiv A \rho^{\sigma} B-B \rho^{\sigma} A,
\end{aligned}
$$

with $\sigma \in[0,1 / 4]$. First, we note that for $0 \leq \alpha \leq 1, C(t ; \beta)_{(\alpha, 0)}$ is positive definite and for $\alpha=\{0,1\}, \sigma=0$ we recover the unregulated thermal commutator-squaredd in the thermal state.

Expanding the terms in $C(t ; \beta)_{(\alpha, \sigma)}$ gives eq. (1.5)

$$
\begin{aligned}
C(t ; \beta)_{(\alpha, \sigma)}= & -\operatorname{Tr}\left[\rho^{1-\alpha-\sigma} W(t) \rho^{\sigma} V \rho^{\alpha-\sigma} W(t) \rho^{\sigma} V+\rho^{1-\alpha-\sigma} V \rho^{\sigma} W(t) \rho^{\alpha-\sigma} V \rho^{\sigma} W(t)\right] \\
& +\operatorname{Tr}\left[\rho^{1-\alpha-\sigma} W(t) \rho^{\sigma} V \rho^{\alpha-\sigma} V \rho^{\sigma} W(t)+\rho^{1-\alpha-\sigma} V \rho^{\sigma} W(t) \rho^{\alpha-\sigma} W(t) \rho^{\sigma} V\right],
\end{aligned}
$$

The last two are conventional Schwinger-Keldysh time-ordered correlation functions (TOCs), whereas the first two are true out-of-time-ordered correlators of the type

$$
\begin{aligned}
F\left(t_{1}, t_{2}\right)_{(\alpha, \sigma)} & \equiv \operatorname{Tr}\left[\rho^{1-\alpha-\sigma} W\left(t_{1}\right) \rho^{\sigma} V \rho^{\alpha-\sigma} W\left(t_{2}\right) \rho^{\sigma} V\right] \\
& =\operatorname{Tr}\left[\rho^{1-\alpha} W\left(t_{1}-i \sigma \beta\right) V \rho^{\alpha} W\left(t_{2}-i \sigma \beta\right) V\right] \\
& =F\left(t_{1}-i \sigma \beta, t_{2}-i \sigma \beta\right)_{(\alpha, 0)} .
\end{aligned}
$$

Schematically $C(t ; \beta)_{(\alpha, \sigma)}$ equals

$$
C(t ; \beta)_{(\alpha, \sigma)}=\operatorname{TOCs}-F(t-i \sigma \beta, t-i \sigma \beta)_{(\alpha, 0)}-F(t-i(1-\alpha-\sigma) \beta, t-i(\alpha-\sigma) \beta)_{(\alpha, 0)} .
$$

Each out-of-time ordered correlator $F\left(t_{1}, t_{2}\right)_{(\alpha, \sigma)}$ may be seen as a correlation function in the extended Schwinger-Keldysh contour. The usual choice with $\alpha=1 / 2, \sigma=1 / 4$ is shown in figure 1-(a); the more general $F\left(t_{1}, t_{2}\right)_{(\alpha, \sigma)}$ corresponds to a more complicated contour like the one shown in figure 1-(b) with different separations in imaginary time between each of the branches.

It is this OTOC $F\left(t_{1}, t_{2}\right)_{(\alpha, \sigma)}$ that controls the regime of exponential growth and the Lyapunov spectrum $F\left(t_{1}, t_{2}\right)_{(\alpha, \sigma)} \sim 1-\mathrm{A}\left(t_{1}-t_{2}\right) e^{\lambda_{(\alpha, \sigma)} \frac{\left(t_{1}+t_{2}\right)}{2}}$, with $\mathrm{A}(0)$ a finite positive number. We will now show that the same exponential time dependence and thus the same Lyapunov exponent is obtained independent of the value of $\sigma$ if $\alpha=1 / 2$. This follows directly from the analyticity property of the function highlighted above: $F\left(t_{1}, t_{2}\right)_{(\alpha, \sigma)}=F\left(t_{1}-i \sigma \beta, t_{2}-i \sigma \beta\right)_{(\alpha, 0)}$ for $0 \leq \sigma \leq \min \{\alpha, 1-\alpha\}$ [3]. Suppose for the particular value $\sigma=0$ the function $F\left(t_{1}, t_{2}\right)_{(\alpha, 0)}$ has the exponential behavior $F\left(t_{1}, t_{2}\right)_{(\alpha, 0)} \simeq \mathrm{A}\left(t_{1}-t_{2}\right) e^{\lambda_{\alpha} \frac{t_{1}+t_{2}}{2}}$ with $\lambda_{\alpha}=\lambda_{(\alpha, 0)}$. Analyticity implies that $F\left(t_{1}+i \xi \beta, t_{2}+i \gamma \beta\right)_{(\alpha, 0)} \simeq \mathrm{A}\left(t_{1}-t_{2}+i \beta(\gamma-\xi \bmod 1)\right) e^{i \frac{\xi+\gamma}{2} \beta \lambda_{\alpha}} e^{\lambda_{\alpha} \frac{t_{1}+t_{2}}{2}}$. Substituting this into eq. (2.4), we get

$$
C(t ; \beta)_{(\alpha, \sigma)} \simeq \mathrm{TOCs}+\left[\mathrm{A}(0) e^{-i \sigma \beta \lambda_{\alpha}}+\mathrm{A}(i \beta(1-2 \alpha \bmod 1)) e^{-i \frac{\beta \lambda_{\alpha}}{2}(1-\alpha-\sigma+\alpha-\sigma)}\right] e^{\lambda_{\alpha} t} .
$$




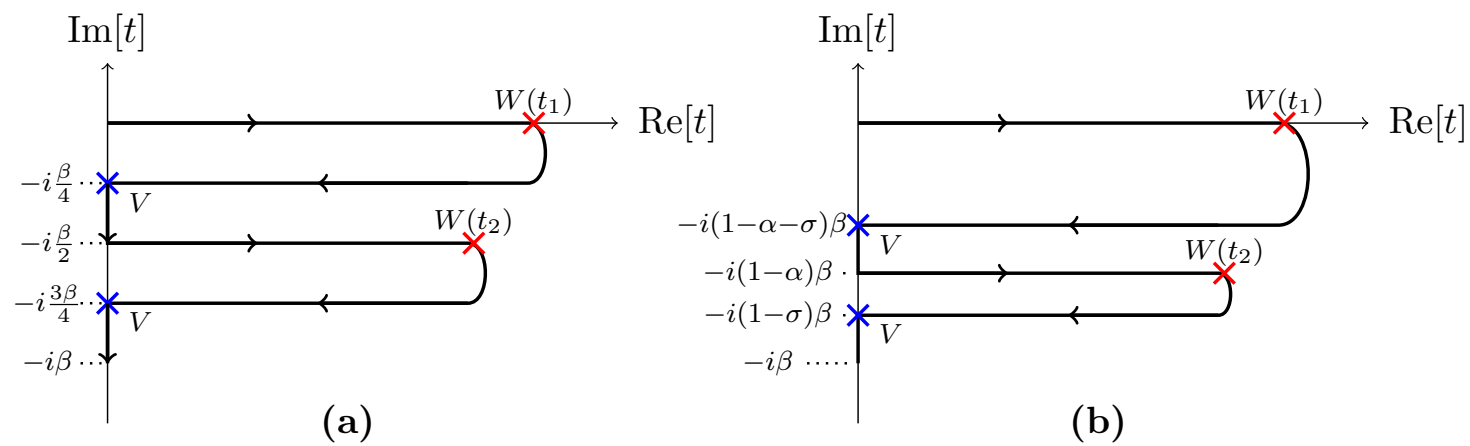

Figure 1. (a) Extended Schwinger-Keldysh contour corresponding to

$\operatorname{Tr}\left[\rho^{\frac{1}{4}} V \rho^{\frac{1}{4}} W\left(t_{2}\right) \rho^{\frac{1}{4}} V \rho^{\frac{1}{4}} W\left(t_{1}\right)\right]$. (b) Contour corresponding to a general regularization of the OTOC $\operatorname{Tr}\left[\rho^{\sigma} V \rho^{\alpha-\sigma} W\left(t_{2}\right) \rho^{\sigma} V \rho^{1-\alpha-\sigma} W\left(t_{1}\right)\right]$, which contributes to $C_{(\alpha, \sigma)}\left(t_{1}, t_{2}\right)$.

For the specific choice $\alpha=1 / 2$ - the one that is made in almost all previous studies the prefactor $\left.\mathrm{A}(i \beta(1-2 \alpha \bmod 1))\right|_{\alpha=1 / 2}=\mathrm{A}(0)$ is the same in both cases and equal to the one computed for the $\alpha=1 / 2$. Thus

$$
C(t ; \beta)_{\left(\frac{1}{2}, \sigma\right)}=\mathrm{TOCs}+2 \mathrm{~A}(0) e^{-i \beta \frac{\lambda_{1 / 2}}{4}} \cos \left[\left(\sigma-\frac{1}{4}\right) \beta \lambda_{1 / 2}\right] e^{\lambda_{1 / 2} t},
$$

with $\lambda_{1 / 2}=\lambda_{\left(\frac{1}{2}, \sigma\right)}, \forall \sigma$. Although the Lyapunov exponent is not affected by the deformation parametrized by $\sigma$ away from $(\alpha, \sigma)=\left(\frac{1}{2}, 0\right)$, we do see that the prefactor of the exponential depends on the $\sigma$-deformation of the contour. Therefore, similarly to a Wightman function in Schwinger-Keldysh theory, the full commutator-squared $C(t ; \beta)_{\left(\frac{1}{2}, \sigma\right)}$ cannot be an observable measurable in an experiment, even though it may contain physical information.

We also point out that the dependence of the prefactor on the contour seems to be in tension with the recent attempts to associate maximal chaos, defined as maximal Lyapunov exponent $\lambda=2 \pi / \beta$, to destructive interference of the commutator-squared $[14,15]$. The destructive interference refers to the fact that, if the decoherence factor equals $\cos (\lambda \beta / 4)$, it vanishes for maximal chaos $\lambda=2 \pi / \beta$. This implies that for maximal chaos the exponential time-dependence should be absent in the symmetric commutator-squared. Our derivation shows, however, that this heavily relies on the analytical continuation chosen. Indeed, the decoherence factor of commutator-squared of eq. (2.6) is $\cos ((1 / 4-\sigma) \lambda \beta)$, which does not vanish for maximal chaos $\lambda=2 \pi / \beta$, provided $0<\sigma \leq 1 / 4$. This casts doubts on how universal the relation between maximal chaos and destructive interference may be.

Moreover, it has also been suggested that in SYK the prefactor of the OTOCs $A \cos (\lambda \beta / 4)$, where $A=\beta J / N$, is an observable which is finite at zero temperature [16]. However, as we have shown above this quantity is contour-dependent and therefore, it is not an obvious physical observable.

To summarize, since the commutator-squared depends on the contour it is not clear whether the regularised commutator-squared is actually an observable. Another possibility may be that not all regularizations of the commutator-squared are physically allowed and 


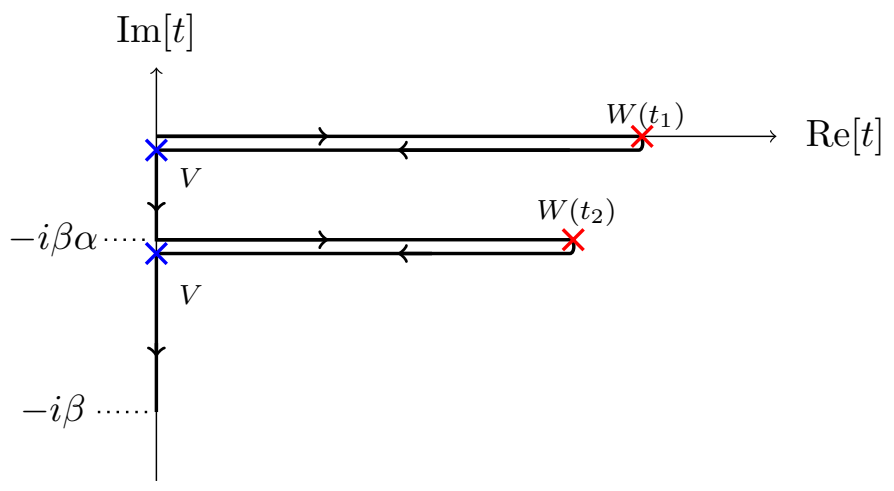

Figure 2. Extended Schwinger-Keldysh contour corresponding to $\operatorname{tr}\left[\rho^{\alpha} W\left(t_{1}\right) V \rho^{1-\alpha} W\left(t_{2}\right) V\right]$ which enters in $C(t ; \beta)_{(\alpha, 0)}$ defined in eq. (2.7).

one value of $\sigma$ is preferred. For the specific deformation parametrized by $\sigma$, we could not find an argument for such case.

\section{$2.1 \quad$ The $\alpha$-contour}

Starting from $\alpha=1 / 2$, the parameter $\sigma$ affects only the decoherence factor of the commutator-squared but leaves the Lyapunov spectrum invariant. There is therefore a possibility that the Lyapunov spectrum as defined through the OTOC does measure a physical quantity. We set $\sigma=0$ from here on and now explore its dependence on the other contour parameter $\alpha$ which fixes the distance between the forwards branches, as shown in figure 2:

$$
C(t ; \beta)_{(\alpha, 0)}=\operatorname{Tr}\left[\mathcal{A}^{\dagger} \mathcal{A}\right] \geq 0, \quad \mathcal{A} \equiv \rho^{\frac{1-\alpha}{2}}[W(t), V(0)] \rho^{\frac{\alpha}{2}}
$$

We have already seen that, for $\alpha \neq \frac{1}{2}$, different choices of $\sigma$ cannot be related by analytic continuation. Neither can $C(t ; \beta)_{(\alpha, 0)}$ and $C(t ; \beta)_{\left(\alpha^{\prime}, 0\right)}$ be related to each other by analytic continuation. In other words, the distance in imaginary time between the forwards branches cannot be compensated by analytic continuation of time. This may be seen explicitly by rewriting the OTOCs in $C(t ; \beta)_{(\alpha, 0)}$ as follows

$$
\begin{aligned}
& \mathcal{H}_{\alpha}\left(t_{1}, t_{2} ; t_{3}, t_{4}\right) \equiv \operatorname{Tr}\left[\rho^{\alpha} V\left(t_{3}\right) W\left(t_{1}\right) \rho^{1-\alpha} V\left(t_{4}\right) W\left(t_{2}\right)\right] \\
& =\operatorname{Tr}\left[V\left(t_{3}+i \beta\left(\alpha-\frac{1}{4}\right)\right) \rho^{\frac{1}{4}} W\left(t_{1}+i \beta\left(\alpha-\frac{1}{2}\right)\right) \rho^{\frac{1}{4}} V\left(t_{4}+i \frac{\beta}{4}\right) \rho^{\frac{1}{4}} W\left(t_{2}\right) \rho^{\frac{1}{4}}\right], \\
& \mathcal{G}_{\alpha}\left(t_{1}, t_{2}\right) \equiv \operatorname{Tr}\left[\rho^{\alpha} W\left(t_{1}\right) V\left(t_{3}\right) \rho^{1-\alpha} W\left(t_{2}\right) V\left(t_{4}\right)\right] \\
& =\operatorname{Tr}\left[W\left(t_{1}+i \beta\left(\alpha-\frac{1}{4}\right)\right) \rho^{\frac{1}{4}} V\left(t_{3}+i \beta\left(\alpha-\frac{1}{2}\right)\right) \rho^{\frac{1}{4}} W\left(t_{2}+i \frac{\beta}{4}\right) \rho^{\frac{1}{4}} V\left(t_{4}\right) \rho^{\frac{1}{4}}\right],
\end{aligned}
$$

where we have chosen to compare to the standard contour with $\rho^{1 / 4}$ separation. The differences between the complexified times, $t_{1}+i \beta\left(\alpha-\frac{1}{2}\right), t_{2}, t_{3}+i \beta\left(\alpha-\frac{1}{4}\right)$ and $t_{4}+i \beta / 4$ in eq. (2.8), no longer vanish in the analytically continued OTOCs and this prevents relating one Lyapunov exponent to another. In particular, the imaginary-time separation between 
the two operators $V(0)$ in both $\mathcal{G}_{\alpha}$ and $\mathcal{H}_{\alpha}$ depends on $\alpha$. The standard choice, $F(t){ }_{\left(\frac{1}{2}, \frac{1}{4}\right)}$, which is the building block used to derive the bound on the Lyapunov exponent [3], is computed on a contour where the separation is $\beta / 2$ and $\alpha=1 / 2$. Therefore, $\mathcal{G}_{\alpha}$ and $\mathcal{H}_{\alpha}$ cannot be related to $F(t)_{\left(\frac{1}{2}, \frac{1}{4}\right)}$ by a simple analytic continuation whenever $\alpha \neq 1 / 2$ and we have to study the behavior of these OTOCs separately.

\subsection{OTOCS and physical observables in SK formalism}

As one may extrapolate from the previous section, the OTOC and its Lyapunov spectrum will in general depend on the Schwinger-Keldysh contour on which it is computed. At first, this result may be surprising because, in standard Schwinger-Keldysh, it is known that physical Green's functions are independent of the contour due to energy conservation $[6,7]$. Indeed, since the doubling of the contour is an artificial mathematical convenience, a priori only correlation functions with external insertions on a single branch should considered physical, e.g.

$$
\left\langle\mathcal{O}^{(1)}\left(x_{1}\right) \mathcal{O}^{(1)}\left(x_{2}\right) \mathcal{O}^{(1)}\left(x_{3}\right)\right\rangle, \quad\left\langle\mathcal{O}^{(2)}\left(x_{1}\right) \mathcal{O}^{(2)}\left(x_{2}\right) \mathcal{O}^{(2)}\left(x_{3}\right) \mathcal{O}^{(2)}\left(x_{4}\right)\right\rangle,
$$

where we indicated with $(i)$ the branch where each operator is inserted. With this definition, the fact that the correlation functions do not depend on the contour is a simple diagrammatic proof. We restate it here for the sake of clarity; it can be found in $[6,7]$.

By inspecting the SK effective action, we know that the interaction vertices are of the form

$$
\mathcal{L}_{\text {int }}=\mathcal{L}_{\text {int }}^{(1)}-\mathcal{L}_{\text {int }}^{(2)}
$$

Consequently, in the diagrammatic expansion each vertex is either of type 1 or of type 2 . The external legs of the vertices are connected to each other or to external operator insertions with the propagators

$$
\left\langle\phi^{(i)}(-k) \phi^{(j)}(k)\right\rangle=\left(\begin{array}{cc}
G_{\text {Feynman }}(k) & G_{\text {Wightman }}^{<}(k) \\
G_{\text {Wightman }}^{>}(k) & G_{\text {anti-Feyman }}(k)
\end{array}\right)_{i j}
$$

Without loss of generality, we focus on the simple lowest order 1PI diagram with $n$ operators inserted the branch 1 and only one $n$-point vertex: $\left\langle\mathcal{O}_{1}^{(1)}\left(k_{1}\right) \ldots \mathcal{O}_{n}^{(1)}\left(k_{n}\right)\right\rangle_{\alpha}$. Clearly if the vertex is of type 1 , there is no contour dependence in the diagram. When the vertex is of type 2 , as in figure 3 , we need to use a Wightman function. For a general contour where the forward and backward branches are separated by $\rho^{\alpha}$ this is one of the Wightman functions ${ }^{2}$

$$
\begin{aligned}
& G_{12}^{\beta \alpha}(k)=\operatorname{Tr} \rho^{1-\alpha} \phi^{(1)}(-k) \rho^{\alpha} \phi^{(2)}(k) \\
& G_{21}^{\beta \alpha}(k)=\operatorname{Tr} \rho^{1-\alpha} \phi^{(2)}(-k) \rho^{\alpha} \phi^{(1)}(k)=e^{\beta(1-2 \alpha) k^{0}} G_{12}^{\beta \alpha}(k)
\end{aligned}
$$

By Fourier transforming the time direction, using $\rho^{\alpha} \hat{O}(t) \rho^{-\alpha}=\hat{O}(t+i \alpha)$ and Fourier transforming back, one readily derives that

$$
\begin{aligned}
& G_{12}^{\beta \alpha}(k)=e^{\beta \alpha k^{0}} G_{12}^{\alpha=0}(k), \\
& G_{21}^{\beta \alpha}(k)=e^{-\beta \alpha k^{0}} G_{21}^{\alpha=0}(k) .
\end{aligned}
$$

\footnotetext{
${ }^{2}$ In the literature the following notation is sometimes used: $G_{12}(k)=G^{<}(k)$ and $G_{21}(k)=G^{>}(k)$.
} 

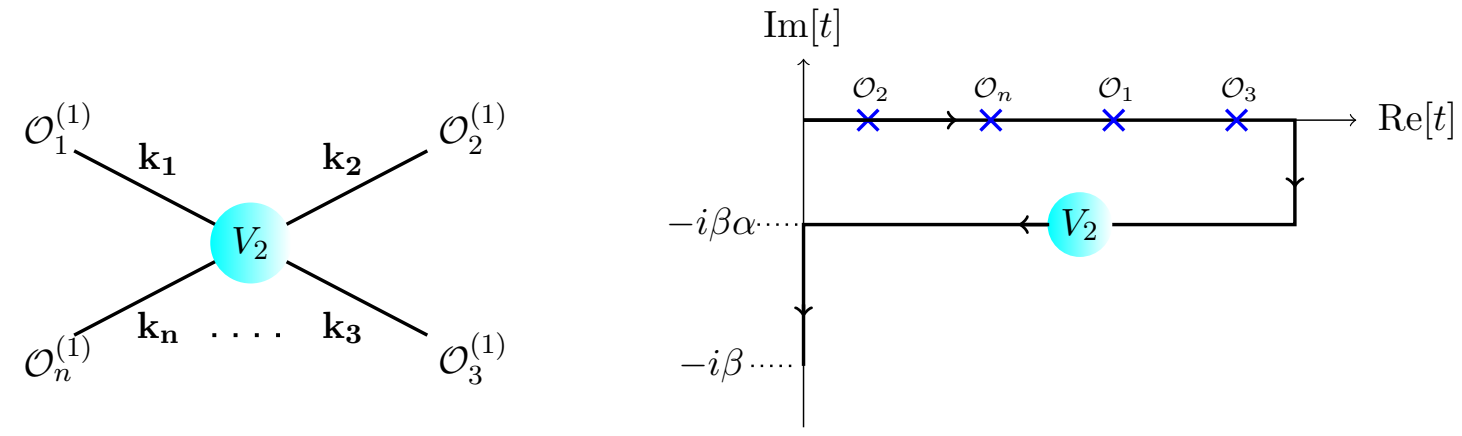

Figure 3. A diagrammatic expansion of the correlator with the external legs on the same branch of the SK contour. The result does not depend on the width $\beta \alpha$.
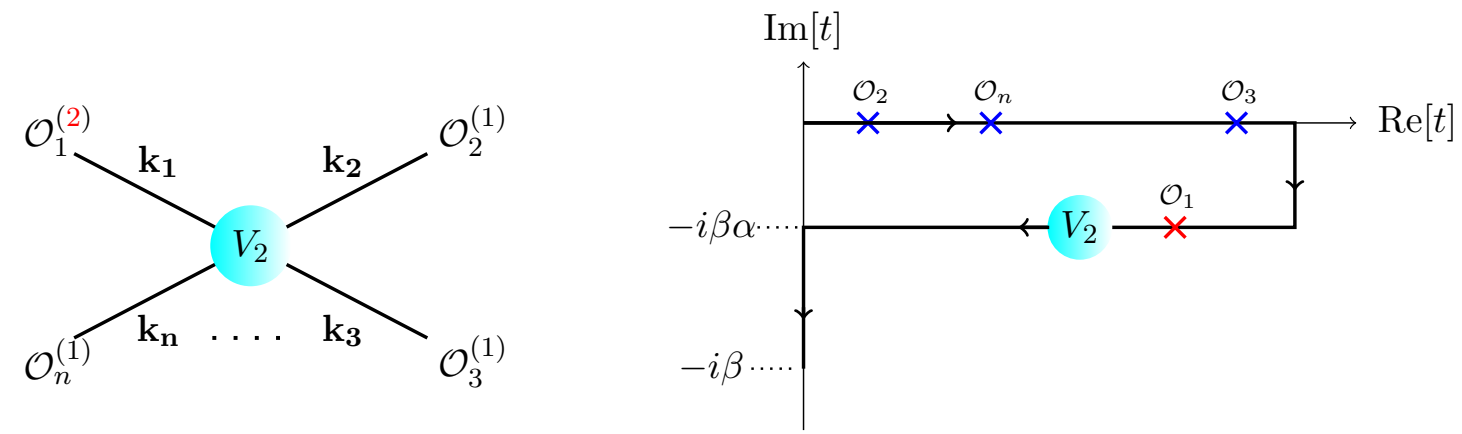

Figure 4. A diagrammatic expansion of the correlator with all but one external legs on the same branch of the SK contour. The result does depend on the width $\beta \alpha$.

At lowest order, there is a single $n$-point vertex on branch 2. Contracting each of the legs of the vertex with the external operators on branch 1 , and by using (2.13), this means that the relation between correlation function on different contours is

$$
\begin{aligned}
\left\langle\mathcal{O}_{1}^{(1)}\left(k_{1}\right) \ldots \mathcal{O}_{n}^{(1)}\left(k_{n}\right)\right\rangle_{\alpha} & \sim e^{\beta \alpha \sum_{i=1, \ldots, n} k_{i}^{0}}\left\langle\mathcal{O}_{1}^{(1)}\left(k_{1}\right) \ldots \mathcal{O}_{n}^{(1)}\left(k_{n}\right)\right\rangle_{\alpha=0} \\
& =\left\langle\mathcal{O}_{1}^{(1)}\left(k_{1}\right) \ldots \mathcal{O}_{n}^{(1)}\left(k_{n}\right)\right\rangle_{\alpha=0}
\end{aligned}
$$

Because of energy conservation at the vertex, $\sum_{i=1, \ldots, n} k_{i}=0$, the overall factor vanishes and this proves the contour independence of these types of diagrams.

However, if one of the external legs is in the branch 2, see figure 4, it is easy to see that now one of the Green's function no longer depends on the separation $\alpha$ at all, so the global factor in the $n$ point function does not simplify anymore. The simplest example of this is the Wightman function itself. There is no vertex, but we have already shown that $G_{12}^{\alpha} \neq G_{12}^{0}$ above in eq. (2.12). Extending to an $n$-point correlation functions with a single $n$-point vertex, one has

$$
\begin{aligned}
\left\langle\mathcal{O}_{1}^{(2)}\left(k_{1}\right) \ldots \mathcal{O}_{n}^{(1)}\left(k_{n}\right)\right\rangle_{\alpha} & \sim e^{\beta \alpha \sum_{i=2, \ldots, n} k_{i}^{0}}\left\langle\mathcal{O}_{1}^{(2)}\left(k_{1}\right) \ldots \mathcal{O}_{n}^{(1)}\left(k_{n}\right)\right\rangle_{\alpha=0} \\
& \neq\left\langle\mathcal{O}_{1}^{(2)}\left(k_{1}\right) \ldots \mathcal{O}^{(1)} n\left(k_{n}\right)\right\rangle_{\alpha=0}
\end{aligned}
$$

but now the exponent in the prefactor $\sum_{i=2, \ldots, n} k_{i}^{0}=-k_{1}^{0} \neq 0$. 
It is not difficult to see that the simple proof shown above extends to any diagrams. Indeed, given any diagram of the expansion, it is sufficient to divide it in subdiagrams and to use the momentum conservation in each vertex.

Turning our attention back to the OTOC, by construction each insertion occurs on one of four different branches. This indicates that the OTOC will be contour dependent, similar to two-branch correlation function in Schwinger-Keldysh theory as depicted in figure 4. If so, this does not immediately mean that the OTOC does not measure a physical quantity (in part). For example, the (bosonic) Wightman function $G_{12}^{\beta \alpha}(k)=e^{\beta k^{0}}\left(1+n\left(k^{0}\right)\right) \rho(k)$ depends on the contour, but still encodes a physical quantity, namely the spectral density $\rho(k)$. Therefore, more care is needed to understand the relation between the contourdependent OTOC and physical properties of the system.

\section{Contour dependence of the Lyapunov spectrum in a matrix $\Phi^{4}$ theory at weak coupling}

We now prove by direct computation that the OTOC indeed depends on the details on the contour chosed. In this section, we compute Lyapunov spectrum obtained from the commutator-squared $C(t ; \beta)_{(\alpha, 0)}$ in a perturbative matrix field theory, which has been studied in detail for $\alpha=1 / 2$ in [8]. The advantage of the perturbative field theory calculation is that the commutator-squared can be related to a kinetic equation encoding the microscopic dynamics [11]. From this, we will suggests that this microscopic insight argues that one specific contour, the one with $\alpha=1 / 2$ is the one that computes microscopic chaos.

We consider a $3+1$ dimensional QFT with a Hermitian matrix field $\Phi_{a b}$ whose Lagrangian is given by

$$
\mathcal{L}=\operatorname{Tr}\left(\frac{1}{2} \dot{\Phi}^{2}-\frac{1}{2}(\nabla \Phi)^{2}-\frac{1}{2} m^{2} \Phi^{2}-\frac{g^{2}}{4 !} \Phi^{4}\right)
$$

with $g^{2}=\lambda N$.

The commutator-squared of eq. (2.7) in this matrix model is

$$
C(t ; \beta)_{(\alpha, 0)}=\frac{1}{N^{4}} \sum_{a b a^{\prime} b^{\prime}} \int d^{3} \mathbf{x} \operatorname{Tr}\left(\rho^{1-\alpha}\left[\Phi_{a b}(t, \mathbf{x}), \Phi_{a^{\prime} b^{\prime}}\right] \rho^{\alpha}\left[\Phi_{a b}(t, \mathbf{x}), \Phi_{a^{\prime} b^{\prime}}\right]^{\dagger}\right) .
$$

For $t>0$, which we shall assume, the lowest order (disconnected) contribution is the product of two retarded Green's function arising from a contraction on the top two folds and the bottom folds separately; there is therefore no contour dependence. The non-trivial contribution at the next order, that can seed exponential growth, is the contribution with two Wightman functions connecting the two retarded Green's functions. For $\alpha=1 / 2$, this equals [8]:

$$
C(\omega)_{\left(\frac{1}{2}, 0\right)}^{(1)}=\frac{1}{N^{2}} \int \frac{\mathrm{d}^{4} p}{(2 \pi)^{4}} \frac{\mathrm{d}^{4} p^{\prime}}{(2 \pi)^{4}} G_{R}(\omega-p) G_{R}(p) R\left(p-p^{\prime}\right) G_{R}\left(\omega-p^{\prime}\right) G_{R}\left(p^{\prime}\right),
$$

where the kernel $R(p)$ is determined in terms of Wightman functions with operators separated by $i \beta / 2$ :

$$
R(p)=\frac{g^{4}\left(N^{2}+5\right)}{12} \int \frac{d^{4} \ell}{(2 \pi)^{4}} G_{12}^{\beta / 2}(p / 2+\ell) G_{12}^{\beta / 2}(p / 2-\ell),
$$


Note that it is only $G_{12}(k)$ and not $G_{21}(k)$, independent of the deformation $\alpha=1 / 2$, which appears inside the kernel. This choice is due to the identity $G_{12}(k)=G_{21}(-k)$. We will consistently use $G_{12}$ only; this will not affect the final result. Defining a function $f(\omega, p)$,

$$
C(\omega)_{\left(\frac{1}{2}, 0\right)}=\frac{1}{N^{2}} \int \frac{d^{4} p}{(2 \pi)^{4}} f(\omega, p),
$$

at the next order one of the contributions is

$$
C(\omega)_{\left(\frac{1}{2}, 0\right)}^{(2)}=\frac{1}{N^{2}} \int \frac{\mathrm{d}^{4} p}{(2 \pi)^{4}} \frac{\mathrm{d}^{4} p^{\prime}}{(2 \pi)^{4}} G_{R}(\omega-p) G_{R}(p) R\left(p-p^{\prime}\right) f^{(1)}\left(\omega, p^{\prime}\right),
$$

and by rewriting $C(\omega)_{\left(\frac{1}{2}, 0\right)}^{(2)}=\frac{1}{N^{2}} \int \frac{d^{4} p}{(2 \pi)^{4}} f^{(2)}(\omega, p)$, one can set up a recursive Bethe-Salpeter equation to determine $f(\omega, p)$ and hence $C(\omega)_{\left(\frac{1}{2}, 0\right)}$ to all orders. Since we are interested in the late-time exponential growth, we focus on the homogeneous part of the Bethe-Salpeter equation, which in the low-frequency, late time limit equals

$$
f(\omega, p) \simeq-G^{R}(p) G^{R}(\omega-p) \int \frac{\mathrm{d}^{4} k}{(2 \pi)^{4}} R(k-p) f(\omega, k),
$$

Equation (3.7) is only appropriate in the low $\omega$ limit. In this limit the product of retarded Green's functions is dominated by a pinching pole singularity, which amounts to the following approximation [8]

$$
G^{R}(p) G^{R}(\omega-p)=\frac{\pi}{E_{\boldsymbol{p}}} \frac{\delta\left(p_{0}^{2}-E_{\boldsymbol{p}}^{2}\right)}{i \omega-2 \Gamma_{p}}+\ldots .
$$

As this concentrates the support of the right hand side of the BSE on the on-shell deltafunction, there is natural ansatz for the solution of $f(\omega, p)$ to be proportional to the same delta-function

$$
f(\omega, p)_{\text {ansatz }}=f(\omega, \boldsymbol{p}) \delta\left(p_{0}^{2}-E_{\boldsymbol{p}}^{2}\right) .
$$

The imaginary part of the two-loop ( $\alpha$-independent) self energy $\Gamma_{p}$ also happens to be determined in terms of (the $\alpha=1 / 2) R(k)$ defined in eq. (3.4):

$$
\Gamma_{\boldsymbol{p}}=\frac{\sinh \left(\frac{\beta E_{\boldsymbol{p}}}{2}\right)}{24 E_{\boldsymbol{p}}} \int \frac{\mathrm{d}^{3} k}{(2 \pi)^{3}} \frac{R\left(E_{\boldsymbol{p}}-E_{\boldsymbol{k}}, \boldsymbol{p}-\boldsymbol{k}\right)+R\left(E_{\boldsymbol{p}}+E_{\boldsymbol{k}}, \boldsymbol{p}-\boldsymbol{k}\right)}{E_{\boldsymbol{k}} \sinh \left(\frac{\beta E_{\boldsymbol{k}}}{2}\right)} .
$$

Including both the pinching pole approximation and the self-energy rewriting in terms of the rung function $R(k-p)$, the low-energy approximation of the BSE reads

$$
-i \omega f(\omega, \boldsymbol{p})=\int \frac{\mathrm{d}^{3} k}{(2 \pi)^{3}}\left(\mathcal{K}(\boldsymbol{p}, \boldsymbol{k})-2 \Gamma_{\boldsymbol{k}}(2 \pi)^{3} \delta^{3}(\boldsymbol{p}-\boldsymbol{k})\right) f(\omega, \boldsymbol{k}),
$$

where $\mathcal{K}(\boldsymbol{p}, \boldsymbol{k})=\frac{R\left(E_{\boldsymbol{p}}-E_{\boldsymbol{k}}, \boldsymbol{p}-\boldsymbol{k}\right)+R\left(E_{\boldsymbol{p}}+E_{\boldsymbol{k}}, \boldsymbol{p}-\boldsymbol{k}\right)}{4 E_{\boldsymbol{k}} E_{\boldsymbol{p}}}$. The positive eigenvalues of the kernel $\mathcal{K}(\boldsymbol{p}, \boldsymbol{k})-2 \Gamma_{\boldsymbol{p}} \delta^{3}(\boldsymbol{p}-\boldsymbol{k})$, considered as a matrix in $k$ and $p$, form the Lyapunov spectrum characterizing the exponential growth at late times, as we will review below. 


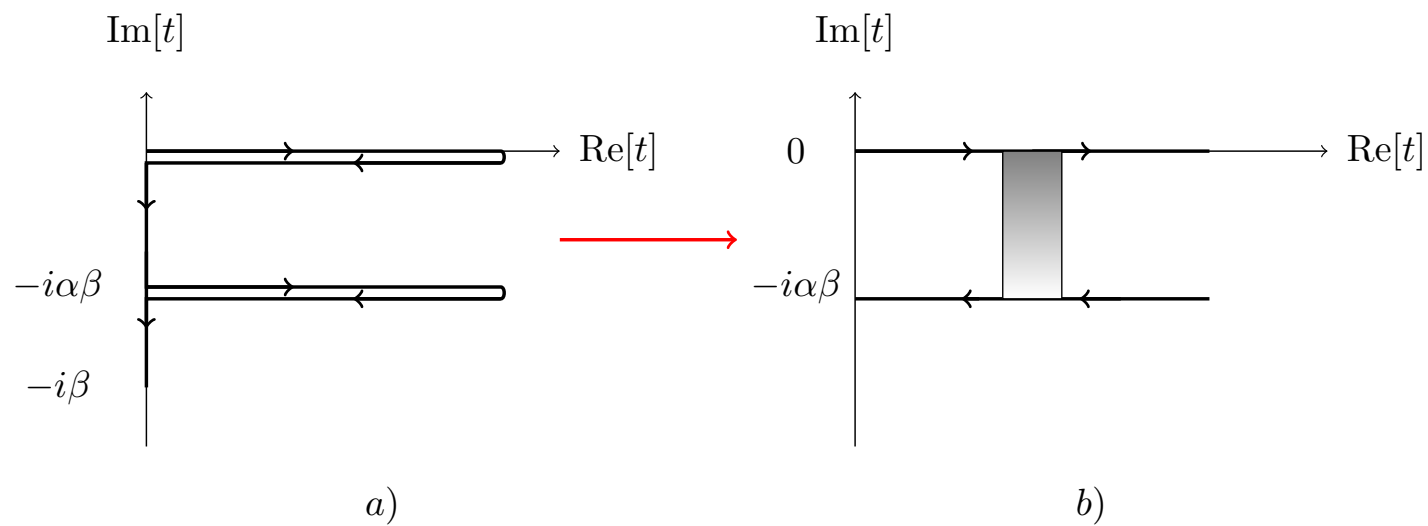

Figure 5. A pictorial representation of a general time contour $(a)$ and of the 4-points function in the ladder approximation $(b)$. The external legs lay on the first time fold and the second time fold. On the contrary, the rung joins the two time folds and include Wightman functions which by definition are contour dependent.

Importantly, the Lyapunov spectrum is not set by the off-shell rung function $R(p-k)$ or the off-shell BSE eq. (3.14) [11]. Specifically, the "on-shell" delta-function ansatz for $f(\omega, p)=f(\omega, \boldsymbol{p}) \delta\left(p_{0}^{2}-E_{p}\right)$, that naturally follows the pinching pole approximation, acts as a projector on the set of functions $f(\omega, p)$. Therefore the set of eigenvalues and eigenvectors of $R(k-p)$ are not the same as those of the kernel in eq. (3.11) which sets the Lyapunov spectrum.

This derivation makes clear that the only $\alpha$-contour-deformation dependence arises from the Wightman functions in the rung function. It is then straightforward to derive the contour-dependence of the OTOC. For $\alpha \neq 1 / 2$, the rung function should be modified as sketched in figure 5. Mathematically

$$
R(p) \rightarrow e^{\beta p^{0}(\alpha-1 / 2)} R(p)
$$

Again, defining

$$
C(\omega ; \beta)_{(\alpha, 0)}=\frac{1}{N^{2}} \int \frac{d^{4} p}{(2 \pi)^{4}} f(\omega, p),
$$

this will now obey the equation:

$$
f(\omega, p) \simeq-G^{R}(p) G^{R}(\omega-p) \int \frac{\mathrm{d}^{4} k}{(2 \pi)^{4}} e^{\eta\left(k^{0}-p^{0}\right)} R(k-p) f(\omega, k),
$$

with $\eta \equiv \beta(\alpha-1 / 2)$. Note that the change in the rung function does not depend on whether it is constructed from $G_{12}(k)$ or $G_{21}(-k)$. This can be confirmed by the fact that the commutator-squared should obey a KMS type symmetry $\alpha \rightarrow 1-\alpha$ on the doubled time contour. This follows by redefining $k \rightarrow \omega-k$ and $p \rightarrow \omega-p$. The kernel $R(k-p)$ is even in $k-p$ as can be readily seen from its definition eq. (3.4). The product $G^{R}(p) G^{R}(\omega-p)$ changes into itself, and one obtains an equation for $f\left(\omega ; \omega-p^{0},-\boldsymbol{p}\right)$ which identical to the original equation. 
To solve the Bethe-Salpeter equation (3.14) after the pinching pole approximation in the late time limit,

$$
f(\omega, p) \simeq \frac{\pi}{E_{\boldsymbol{p}}} \frac{\delta\left(p_{0}^{2}-E_{\boldsymbol{p}}^{2}\right)}{-i \omega+2 \Gamma_{p}} \int \frac{\mathrm{d}^{4} k}{(2 \pi)^{4}} e^{\eta\left(k^{0}-p^{0}\right)} R(k-p) f(\omega, k),
$$

one then makes the natural ansatz

$$
f(\omega, p)=f(\omega, \boldsymbol{p}) \delta\left(p_{0}^{2}-E_{\boldsymbol{p}}^{2}\right)
$$

However, note that the choice of the ansatz is very subtle and might lead to a different physical solution. By inspecting eq. (3.14), one might be tempted to argue that, since the $\eta$ dependent term resembles a similarity transformation, the eigenvalues are unchanged. This conclusion is not correct. Indeed, as briefly recalled above, we showed in [11] that there are physical implications in this choice. Most notably, the $\eta=0$ BSE with the kernel replacement $R(k-p) \rightarrow \frac{\left.\sinh \left(\beta p_{0} / 2\right)\right)}{\sinh \left(\beta k_{0} / 2\right)} R(k-p)$ corresponds to the evaluation of a different analytic continuation of the commutator-squared. This analytic continuation is the retarded correlation function of the Wigner transform of the bilocal density operator, namely the correlation function that appears in the Kubo formula of the shear viscosity. From Boltzmann's kinetic theory, the spectrum with this ansatz is manifestly negative definite (i.e. there are only decaying modes). In other words, the choice of contour dictates the ansatz to solve the BSE. At the same time, all the contours that are related through analytic continuation arise from the same contour-independent off shell BSE eq. (3.15).

Substituting this appropriate ansatz (3.16) into eq. (3.14), we then perform the integral over $p_{0}$. This yields

$$
\left(-i \omega+2 \Gamma_{p}\right) f(\omega, \boldsymbol{p})=\int \frac{\mathrm{d}^{3} k}{(2 \pi)^{3}} f(\omega, \boldsymbol{k}) \mathcal{K}(\boldsymbol{k}, \boldsymbol{p}, \eta)
$$

with

$\mathcal{K}(\boldsymbol{k}, \boldsymbol{p}, \eta) \equiv \frac{\cosh \left[\eta\left(E_{\boldsymbol{p}}-E_{\boldsymbol{k}}\right)\right] R\left(E_{\boldsymbol{p}}-E_{\boldsymbol{k}}, \boldsymbol{p}-\boldsymbol{k}\right)+\cosh \left[\eta\left(E_{\boldsymbol{p}}+E_{\boldsymbol{k}}\right)\right] R\left(E_{\boldsymbol{p}}+E_{\boldsymbol{k}}, \boldsymbol{p}-\boldsymbol{k}\right)}{4 E_{\boldsymbol{k}} E_{\boldsymbol{p}}}$,

where we have explicitly used that the rung kernel is even in the energy argument: $R\left(k_{0}, \boldsymbol{k}\right)=R\left(-k_{0}, \boldsymbol{k}\right)$. Substituting eq. (3.10) into eq. (3.17), we arrive at the final BetheSalpeter equation for $C(\omega)_{(\alpha, 0)}=\frac{1}{N^{2}} \int \frac{d^{4} p}{(2 \pi)^{4}} f(\omega, p)$ in the frequency domain:

$$
-i \omega f(\omega, \boldsymbol{p})=\int \frac{\mathrm{d}^{3} k}{(2 \pi)^{3}}\left[f(\omega, \boldsymbol{k}) \mathcal{K}(\boldsymbol{k}, \boldsymbol{p}, \eta)-f(\omega, \boldsymbol{p}) \frac{\sinh \left(\frac{\beta E_{\boldsymbol{p}}}{2}\right)}{\sinh \left(\frac{\beta E_{\boldsymbol{k}}}{2}\right)} \frac{R\left(E_{-}, \boldsymbol{p}-\boldsymbol{k}\right)+R\left(E_{+}, \boldsymbol{p}-\boldsymbol{k}\right)}{12 E_{\boldsymbol{p}} E_{\boldsymbol{k}}}\right],
$$

where $E_{ \pm} \equiv E_{\boldsymbol{p}} \pm E_{\boldsymbol{k}}$. In the time domain this is an equation of the type

$$
\frac{\partial}{\partial t} f(t)_{p}=\mathcal{M}_{\boldsymbol{p} \boldsymbol{k}} f_{\boldsymbol{k}}(t)
$$



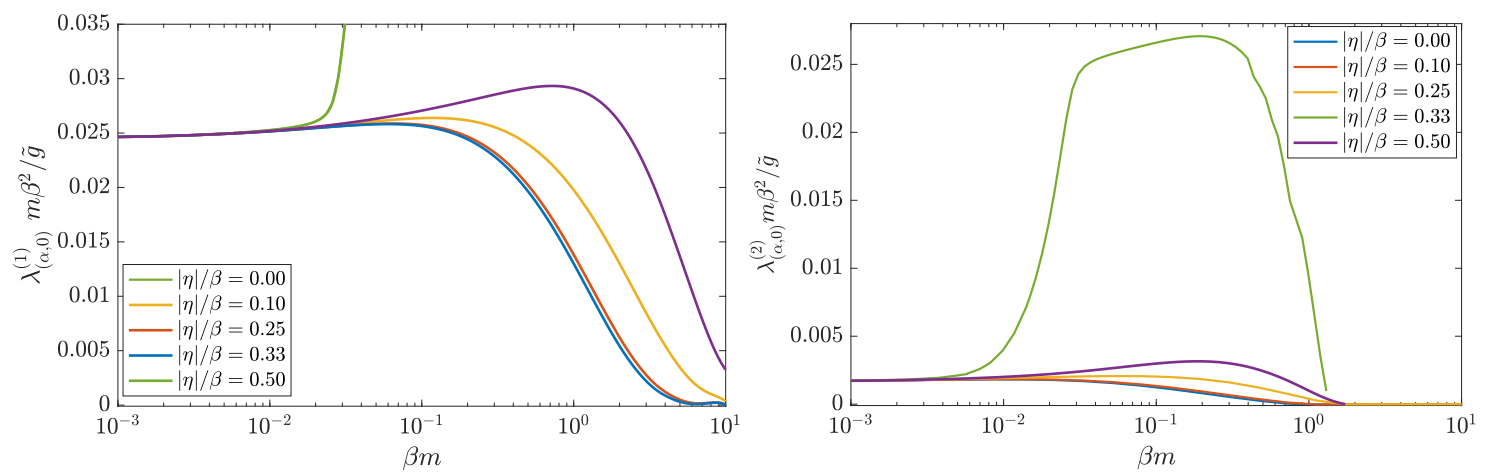

Figure 6. Contour dependence of the Lyapunov spectrum in the weakly coupled $\Phi^{4}$-matrix model. Two largest Lyapunov exponents of Lyapunov spectrum of the matrix $\Phi^{4}$ theory as a function of the coupling. Each value of $\eta$, defined in eq. (3.14), corresponds to a different contour choice: $\eta=0$ corresponds to the common symmetric regularization. For brevity, we defined $\tilde{g}=\frac{g^{4}\left(N^{2}+5\right)}{4 \cdot 144}$.

The solutions are the eigenvectors of $\mathcal{M}_{\boldsymbol{p} \boldsymbol{k}}$ with an exponential growth/decay in time proportional to the eigenvalue. The positive eigenvalues of $\mathcal{M}_{p k}$ are the Lyapunov spectrum. This can be found numerically; the precise method used to solve this equation may be found in appendix A. Without computation it is already clear, however, that the result will depend on the $\alpha$-deformed contour, as the defining Bethe-Salpeter equation does so.

The result is presented in figure 6 . We clearly see the dependence of the two positive Lyapunov exponents on the contour. The spectrum does become contour-independent in the high-temperature limit. This follows directly from the fact that the deformation parametrized by $\eta=\beta(\alpha-1 / 2)$ becomes negligible for small $\beta$ (compared to the mass).

That in these models the Lyapunov spectrum is contour independent for zero mass, will be crucial to understand the SYK model, which we study in the next section. There, there are only gapless excitations and not even a thermal mass, and we can therefore expect the same contour independence of the Lyapunov spectrum as the $\beta m \rightarrow 0$ limit of weakly coupled field theories as exhibited in figure 6. Do recall that the full OTOC always depends on the contour.

For intermediate and small $\beta$, the Lyapunov spectrum sensitively depends on the choice of contour. As also noted already in [8], in the extreme low temperature limit $\beta m \rightarrow \infty$, the Lyapunov spectrum vanishes exponentially in $\beta m$. Even though this decreases the relative dependence on the contour, the contour dependence still persists and is given by $e^{-(\beta+2|\eta|) m}$, as shown in figure 7 .

Let us make one final comment on the connection between the choice of ansatz and the contour dependence of the Lyapunov spectrum. One readily observes that another possible ansatz to the BSE is

$$
\begin{aligned}
f(\omega, p) & =f(\omega, \boldsymbol{p}) e^{\eta p^{0}} \delta\left(p_{0}^{2}-E_{\boldsymbol{p}}^{2}\right) \\
& =f(\omega, \boldsymbol{p})\left(e^{\eta E_{\boldsymbol{p}}} \frac{\delta\left(p^{0}-E_{\boldsymbol{p}}\right)}{2 E_{\boldsymbol{p}}}+e^{-\eta E_{\boldsymbol{p}}} \frac{\delta\left(p^{0}+E_{\boldsymbol{p}}\right)}{2 E_{\boldsymbol{p}}}\right) .
\end{aligned}
$$

This $\eta$-contour skewed ansatz gives a contour independent Lyapunov exponent as solution 


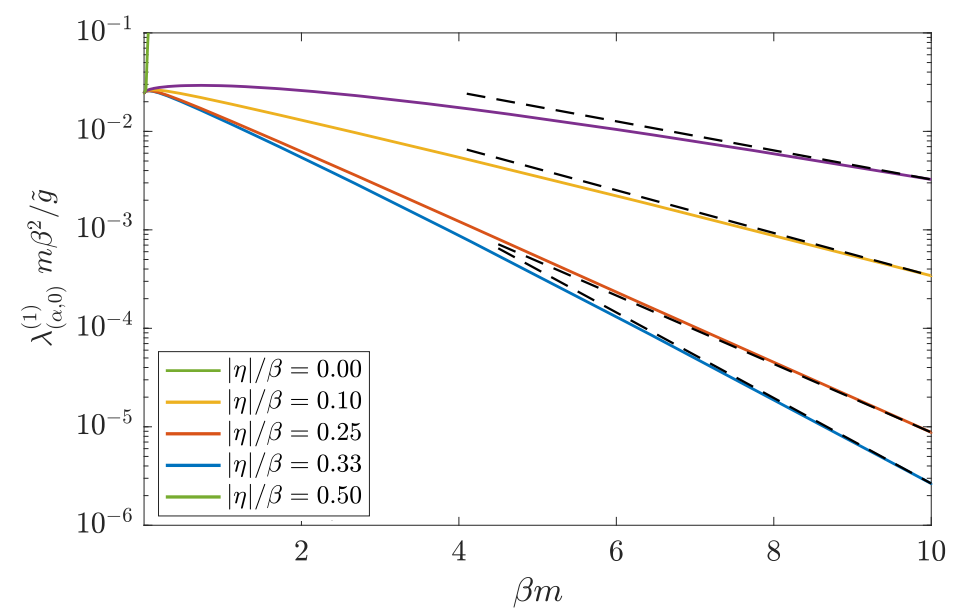

Figure 7. Exponential decay of the first Lyapunov exponent for various contours. Dashed lines correspond to the analytical expression $C e^{-(\beta+2|\eta|) m}$, where $C$ is fixed so that the dashed line passes through the last point available $\beta m=10$. For brevity, we defined $\tilde{g}=\frac{g^{4}\left(N^{2}+5\right)}{4 \cdot 144}$.

for eq. (3.15) and coincides with the solution for $\eta=0$. One may ask why one ansatz is preferred over the other. As is clear from figure 6, the natural ansatz (3.16) represents a solution with a larger eigenvalue of the Lyapunov exponent. We therefore argue that this solution is what a general computation, i.e. using different techniques than the BSE, of the leading exponential rate of growth in the OTOC would capture. In support of this, we also refer to the results of [5]. There, the authors computed the OTOC for a $2+1$ disordered systems by means of a Keldysh nonlinear sigma model technique that they developed. Within this framework, the computation of the largest exponent for the unregularised $\eta=1 / 2$ case and the regularised $\eta=0$ case gives a different result. Moreover, the unregularised case has a larger exponetial growth rate. The explanation is the one we give above.

This is the message to take from these results. When one computes the OTOC, one is inherently concerned with the late time regime of the correlator and with the largest term in the exponential growth. Mathematically the Lyapunov exponent of the fastest growing mode is contour dependent. This gives rise to the physics question of how we can understand the different contour dependent growing rates. We will answer this question in section 3.2 .

\subsection{The contour dependence regulates the IR}

The contour dependence of the Lyapunov spectrum explicitly exhibited above emphasizes an important point regarding the physics behind the contour deformation. One of the arguments made for deforming contour symmetrically

$$
C(t ; \beta)_{\text {regulated }}=\operatorname{Tr}\left(\rho^{\frac{1}{2}}[W(t), V]^{\dagger} \rho^{\frac{1}{2}}[W(t), V]\right),
$$

or

$$
F(t, t)_{\left(\frac{1}{2}, \frac{1}{4}\right)}=\operatorname{Tr}\left(\rho^{\frac{1}{4}} W(t) \rho^{\frac{1}{4}} V \rho^{\frac{1}{4}} W(t) \rho^{\frac{1}{4}} V\right)
$$


is that the smearing of the density matrix regulates a short distance singularity by separating the local operators in imaginary time. If this were indeed what the smearing should accomplish, then (1) at any finite value of regulator $\eta$ we should expect the lowtemperature limit to be universal, and (2) at any finite temperature $\beta$ in units of the mass $m$ the answer for the OTOC should diverge as one removes the regulator $|\eta| \rightarrow \frac{\beta}{2}$. The result, however, shows the opposite. The high-temperature limit is universal, indicating that this is the regime that is insensitive to the regulator, and, though we do not compute the full OTOC, the Lyapunov spectrum at fixed $\beta m$ stays finite for any value of regulator. This argues strongly that the contour-deformation regulates the IR rather than the UV. This in fact agrees with Schwinger-Keldysh theory. There, the "contour-deformation" is the introduction of temperature itself, and this is a well-known IR regulator.

For results in the literature in perturbative QFTs, this diametrically opposite interpretation of the contour deformation has little effect. As in e.g $[8,11,17,18]$ usually the focus is on the universal high temperature regime. However, for the SYK model, the focus has often been on the emergent regime at low temperatures. There, this realization that the contour deformation regulates the IR may imply that the results are in fact regulator dependent and do not reflect physical information about the true dynamics. As we will show in section 4, SYK is special in that its gapless nature and the absence of a thermal mass imply contour independence of the Lyapunov spectrum even at low temperature, extending from the $\beta m \rightarrow 0$ regime of weakly coupled field theories. Before we turn to this, we first address how to obtain the physical information about the true chaos/scrambling dynamics at low temperatures.

\subsection{Kinetic theory interpretation of the $\alpha$-deformed OTOC}

IR regulators often encode real physical circumstances. The correct question to ask therefore is which contour properly reflects physical information of microscopic chaos. In this section we will argue that this can be decided by interpreting the result of the previous section in terms of the kinetic theory for many body chaos derived in [11]. There, the authors showed that the computation of the $\alpha=1 / 2, \sigma=1 / 4$ OTOC is equivalent to a Boltzmann-like equation that tracks the time evolution of the gross energy exchange.

We briefly review this result. The standard Boltzmann equation describes the time evolution of the single-particle distribution function $f(t, \mathbf{r}, \mathbf{p}),{ }^{3}$ parametrizing the deviation of the single-particle distribution function from its equilibrium value:

$$
f(t, \boldsymbol{p})=\frac{\delta n(t, \boldsymbol{p})}{(1+n(\boldsymbol{p})) n(\boldsymbol{p})},
$$

and $n(\boldsymbol{p})$ is the Bose-Einstein distribution. For small deviations from the equilibrium value, the Boltzmann equation can be linearized and, focusing on the homogeneous case, it reads

$$
\partial_{t} f(t, \boldsymbol{p})=-\int_{\boldsymbol{l}} \mathcal{L}(\mathbf{p}, \mathbf{l}) f(t, \boldsymbol{l}),
$$

\footnotetext{
${ }^{3}$ Not to be confused with the commutator-squared function defined in eq. (3.13).
} 
where $\mathcal{L}(\mathbf{p}, \mathbf{l})$ represents the collision integral. $\mathcal{L}(\mathbf{p}, \mathbf{l})$ contains two contributions, namely the gain term $R^{\wedge}(\mathbf{p}, \mathbf{l})$, counting increase of the density of the phase-space cell, and the loss term $R^{\vee}(\mathbf{p}, \mathbf{l})$, which accounts for scattering out of the phase-space cell. In terms of these two contributions, the Boltzmann equation is

$$
\partial_{t} f(t, \boldsymbol{p})=\int_{\boldsymbol{l}}\left[R^{\wedge}(\mathbf{p}, \boldsymbol{l})-R^{\vee}(\mathbf{p}, \boldsymbol{l})\right] f(t, \boldsymbol{l}) .
$$

As shown in [11], the Bethe-Salpeter equation of the symmetrised commutator-squared $C(t ; \beta)_{\left(\frac{1}{2}, 0\right)}$ is equivalent to considering a Boltzman-like equation where the sign of the contribution of the true loss term is changed, so that we account for a gross exchange rather than a net exchange. More precisely, the gross exchange is given by

$$
\partial_{t} f^{\mathrm{EX}}(t, \boldsymbol{p})=\int_{\boldsymbol{l}} \frac{\mathcal{E}\left[E_{\boldsymbol{p}}\right]}{\mathcal{E}\left[E_{\mathbf{l}}\right]}\left[R^{\wedge}(\mathbf{p}, \boldsymbol{l})+R^{\vee}(\mathbf{p}, \boldsymbol{l})-4 \Gamma_{\boldsymbol{l}} \delta(\mathbf{p}-\boldsymbol{l})\right] f^{\mathrm{EX}}(t, \boldsymbol{l}),
$$

where $\mathcal{E}\left[E_{\boldsymbol{p}}\right]=1 / \sinh \left(E_{\boldsymbol{p}} \beta / 2\right)$ is an energy-related observable which does not alter the spectrum of the collision integral, as it enters in the form of a similarity transformation. The extra factor $\Gamma_{l}$, the self-energy due to the thermal environment, is present to avoid over-counting. It can be understood as follows: $R^{\vee_{T}}(\mathbf{p}, \mathbf{l}) \equiv R^{\vee}(\mathbf{p}, \boldsymbol{l})-2 \Gamma_{\mathbf{l}} \delta(\mathbf{p}-\mathbf{l})$ counts the changes in the particle number $f(t, \mathbf{p})$ due only to processes with $\mathbf{p} \neq \boldsymbol{l}$. Therefore, changing the sign of $R^{\vee}$ in eq. (3.25) would over-count the contribution from the bath. If one changes only the sign of the true loss term $R^{\vee_{T}}(\mathbf{p}, \mathbf{l})$, the gross exchange is exactly given by $R^{\wedge}(\mathbf{p}, \boldsymbol{l})+R^{\vee}(\mathbf{p}, \boldsymbol{l})-4 \Gamma_{\boldsymbol{l}} \delta(\mathbf{p}-\boldsymbol{l})[11]$. The eigenvalues of the integral operator (3.26) are equivalent to those measuring the exponential growth rate of the OTOC, and thus give the Lyapunov spectrum of the theory.

As the $\alpha$-deformation only changes the rung function in the Bethe-Salpeter equation, resulting in result (3.18), it is immediately recognized that the kinetic equation encoding the late time behavior of these families of OTOC is modified as follows

$$
\begin{aligned}
\partial_{t} f^{\mathrm{EX}}(t, \boldsymbol{p})= & \int_{\mathbf{l}} \frac{\mathcal{E}\left[E_{\mathbf{p}}\right]}{\mathcal{E}\left[E_{\mathbf{l}}\right]}\left\{\cosh \left[\eta\left(E_{\boldsymbol{p}}-E_{\mathbf{l}}\right)\right] R^{\wedge}(\mathbf{p}, \mathbf{l})\right. \\
& +\cosh \left[\eta\left(E_{\boldsymbol{p}}+E_{\mathbf{l}}\right)\right] R^{\vee}(\mathbf{p}, \mathbf{l}) \\
& \left.-2\left[\cosh \left[2 \eta\left(E_{\boldsymbol{p}}\right)\right]+1\right] \Gamma_{\mathbf{l}} \delta(\mathbf{p}-\mathbf{l})\right\} f^{\mathrm{EX}}(t, \boldsymbol{l}) .
\end{aligned}
$$

The kinetic equation equivalent of the contour-dependent commutator square gives us a direct physical interpretation of what is computed, as we understand each term as loss, gain and self-energy terms in the microscopic dynamics. The explicit $\eta=\beta(\alpha-1 / 2)$ dependence in eq. (3.27) shows that the different contours in the $\alpha$-family have a different physical origin. While for $\eta=0$ (symmetric regularization) both the gain and loss processes are weighted equally, for other contours $\eta \neq 0$, their relative weight is different. For none of these values does the kinetic equation have an obvious natural physical interpretation in terms of gross, net or otherwise simple exchange dynamics.

On the other hand, the gross exchange equation has been put forward independently already a long time ago as a measure of microscopic classical chaos [13]. This conclusion 
from the weakly coupled field theory computation above therefore strongly suggests that, in order to probe dynamical many-body chaos in QFT, the correct choice for the out-of-time correlation function is the symmetrically regularized choice with $\eta=0$. Fortuitously, this is the one that has predominated all the calculations in the literature, including the derivation of the MSS bound on chaos [3]. It also means that the naive thermal expectation value of the commutator-squared $\operatorname{Tr}\left[\rho[W(t), V]^{2}\right]$ does not measure microscopic quantum chaos. One is therefore left with the reversed question: how does one justify from first principles the symmetrically regularized commutator-squared as a measure of quantum chaos. We will return to this question in the last section. First, we will consider the same question of contour-dependence of the commutator-squared and its Lyapunov spectrum for the case of the SYK model.

\section{Contour dependence of the Lyapunov exponent in the SYK model}

One of the research directions where the commutator-squared has had important impact is in the emergent strongly coupled low energy regime of the Sachdev-Ye-Kitaev model. The exponential growth of the symmetrically regularized commutator-squared saturates the MSS bound on chaos $\lambda_{L} \leq 2 \pi T$; this has given great impetus to the notion that the SYK model provides a microscopic theory for AdS black holes.

Now that we know that the commutator-squared and its Lyapunov spectrum depend on the way the contour is regulated, the natural question on how this affects the insights in the SYK model arises. We shall first show that, in contrast to the previous weakly coupled massive QFT results, in the SYK model the Lyapunov spectrum is contour regularization independent.

The SYK Hamiltonian with $q / 2$-body interactions is

$$
H=i_{1 \leq i_{1}<i_{2}<\cdots<i_{q} \leq N} J_{i_{1}, i_{2}, \ldots, i_{q}} \chi_{i_{1}} \chi_{i_{2}} \ldots \chi_{i_{q}}
$$

where $\chi_{i}$ are Majorana fermions so $\left\{\chi_{i}, \chi_{j}\right\}=\delta_{i j}$ and the coupling $J_{i_{1}, i_{2}, \ldots, i_{q}}$ is a Gaussiandistributed random variable with zero average and diagonal (i.e. for each $J_{i_{1}, i_{2}, \ldots, i_{q}}$ independently) variance $\frac{2^{q-1}}{q} \frac{J^{2}(q-1) !}{N^{q-1}}[19]$. The fermionic two-point function $G(\tau)=-\langle\mathcal{T} \chi(\tau) \chi(0)\rangle$ satisfies the following averaged Dyson equation in the large- $N$ limit [19]:

$$
G_{n}^{-1}=-i \omega_{n}-\Sigma_{n}, \Sigma(\tau)=-J^{2} G(\tau)^{q-2} G(-\tau),
$$

with $\omega_{n}=(2 \pi / \beta)(n+1 / 2), G_{n} \equiv G\left(i \omega_{n}\right)$ and $\Sigma_{n} \equiv \Sigma\left(i \omega_{n}\right)$. In the same way as for weakly coupled QFT, the symmetrical contour regularized commutator-squared $C(t ; \beta)_{\left(\frac{1}{2}, 0\right)}$ satisfies a Bethe-Salpeter equation. In the large- $N$ limit, for arbitrary coupling, the homogeneous part of the BS-equation governs the large time limit and is [19]:

$$
F\left(t_{1}, t_{2}\right)=J^{2}(q-1) \int \mathrm{d} t_{3} \mathrm{~d} t_{4} G^{R}\left(t_{13}\right) G^{R}\left(t_{24}\right)\left[G^{W}\left(t_{34}\right)\right]^{q-2} F\left(t_{3}, t_{4}\right),
$$

where $G^{R}$ and $G^{W}$ are the retarded and Wightman two-point functions. 
There is now a difference with the the perturbative QFT approach. As recalled in the previous section, there the late time approximation also involves a pinching pole "onshell" reduction of the retarded Green's functions. The large $N$ late time limit in SYK, on the other hand, is a conformal field theory with no on-shell particle-like excitations. There is no natural simplification of the retarded SYK Green's functions in this limit. In contrast to the perturbative QFT solution, the full large $N$ Green's functions are obtained by analytically continuing the Dyson equation eq. (4.2) to real time and solving these equations numerically with an iterative procedure [20]. ${ }^{4}$ Then one solves the SYK BSE eq. (4.3) by making the explicit ansatz $F\left(t_{1}, t_{2}\right)=e^{\lambda_{L}\left(t_{1}+t_{2}\right) / 2} f\left(t_{12}\right)$ and rewriting it as an integral eigenvalue equation in frequency space:

$$
\begin{aligned}
f\left(\omega^{\prime}\right) & =(q-1) J^{2}\left|G^{R}\left(\omega^{\prime}+i \frac{\lambda_{L}}{2}\right)\right|^{2} \int \frac{\mathrm{d} \omega}{2 \pi} g_{l r}\left(\omega^{\prime}-\omega\right) f(\omega), \\
g_{l r}(\omega) & \equiv \int \mathrm{d} t e^{i \omega t} G^{W}(t)^{q-2} .
\end{aligned}
$$

One finally (numerically or analytically) searches for which value of $\lambda_{L}$ the kernel has an eigenvector with eigenvalue 1 [19].

We can now ask how the subtly different SYK computation of its Lyapunov spectrum depends on the contour. As in the perturbative QFT of the section 3, the only place the contour regularization shows up is in the Wightman functions. ${ }^{5}$ Instead of parametrizing with respect to the $\alpha=0$ Wightman function, let us parametrize with respect to the $\alpha=1 / 2$ Wightman function:

$$
G^{\eta}(\omega)=e^{\eta \omega} G^{\eta=0}(\omega) .
$$

The Bethe-Salpeter equation (4.3) for the commutator square in frequency space for arbitrary $\alpha$-deformed contour is then the same as before, but with a modified kernel $\tilde{g}_{l r}(\omega)$ :

$$
\begin{aligned}
\tilde{f}\left(\omega^{\prime}\right) & =(q-1) J^{2}\left|G^{R}\left(\omega^{\prime}+i \frac{\lambda_{\alpha}}{2}\right)\right|^{2} \int \frac{\mathrm{d} \omega}{2 \pi} \tilde{g}_{l r}\left(\omega^{\prime}-\omega\right) \tilde{f}(\omega), \\
\tilde{g}_{l r}(\omega) & \equiv \int \mathrm{d} t e^{i \omega t} G^{\eta}(t)^{q-2}, \quad G^{\eta}(t)=\int \mathrm{d} \omega e^{-i \omega t} G^{\eta}(\omega) .
\end{aligned}
$$

We evaluate the modification in the kernel $\tilde{g}_{l r}(\omega)$, compared to the original kernel $g_{l r}(\omega)$, by using the convolution of the Wightman functions:

$$
\tilde{g}_{l r}(\omega)=\int \mathrm{d} t e^{i \omega t} G^{\eta}(t)^{q-2}=\int \mathrm{d} \omega_{1} \ldots \mathrm{d} \omega_{q-3} G^{\eta}\left(\omega_{1}\right) G^{\eta}\left(\omega_{2}\right) \ldots G^{\eta}\left(\omega-\omega_{1}-\cdots-\omega_{q-3}\right),
$$

and substituting $G^{\eta}(\omega)=e^{\omega \eta} G^{W}(\omega)$ in each term inside the integral:

$$
\begin{aligned}
\tilde{g}_{l r}(\omega) & =\int_{\omega_{1}, \ldots, \omega_{q-3}} e^{\eta \omega_{1}} G^{\eta}\left(\omega_{1}\right) e^{\eta \omega_{2}} G^{\eta}\left(\omega_{2}\right) \ldots e^{\eta\left(\omega-\omega_{1}-\cdots-\omega_{q-3}\right)} G^{\eta}\left(\omega-\omega_{1}-\cdots-\omega_{q-3}\right) \\
& =e^{\eta \omega} \int \mathrm{d} t e^{i \omega t} G^{W}(t)^{q-2}=e^{\eta \omega} g_{l r}(\omega) .
\end{aligned}
$$

\footnotetext{
${ }^{4}$ As we are using the symmetric regularization, $G^{W}(\omega)=\rho(\omega) \frac{e^{-\omega \frac{\beta}{2}}}{1+e^{-\beta \omega}}$ is the Wightman function with operators separated by $i \beta / 2$.

${ }^{5}$ This can also be seen explicitly by analytically continuing eq. (4.3) $t_{1} \rightarrow t_{1}+i \beta\left(\alpha-\frac{1}{4}\right), t_{2} \rightarrow$ $t_{2}+i \frac{\beta}{4}, t_{3} \rightarrow t_{3}+i \beta\left(\alpha-\frac{1}{2}\right), t_{4} \rightarrow t_{4}$.
} 
Therefore, eq. (4.6) reduces to

$$
\tilde{f}\left(\omega^{\prime}\right)=(q-1) J^{2}\left|G^{R}\left(\omega^{\prime}+i \frac{\lambda_{\alpha}}{2}\right)\right|^{2} \int \frac{\mathrm{d} \omega}{2 \pi} e^{\eta\left(\omega^{\prime}-\omega\right)} g_{l r}\left(\omega^{\prime}-\omega\right) \tilde{f}(\omega),
$$

The crucial difference with weakly coupled QFT is that, because of the gapless nature of SYK even at finite temperature and the absence of a pinched pole on-shell condition, the product of retarded Green's functions remains a smooth function and not a distribution. This allows one to reabsorb the contour dependence with the redefinition $\tilde{f}\left(\omega^{\prime}\right) \rightarrow \tilde{e}^{-\eta \omega^{\prime}} f\left(\omega^{\prime}\right) .{ }^{6}$ In the late time SYK-BSE, this acts as a similarity transformation on the kernel, and its eigenvalues equal to its Lyapunov spectrum is therefore contour independent. Note again that the OTOC is still contour dependent, yet the Lyapunov exponent is independent of the regularization. As mentioned before, this can be qualitatively understood from the weakly coupled case, where in the massless case the dependence of the contour on the Lyapunov exponent vanishes.

To check the solution obtained with the BSE, we now consider the two regimes of the SYK model where some analytical control is possible: the strong coupling limit $\beta J \gg 1$ and the large- $q$ limit.

\subsection{Study of the OTOC in SYK in the strongly coupled limit: conformal limit analysis}

In the strongly-coupled regime $\beta J \gg 1$ of the SYK model, where conformal symmetry emerges asymptotically, the OTOC may also be computed analytically by studying the spectrum of the Casimir operator. More specifcally, for $\beta J \gg 1$ the eigenvectors of the Casimir operator, with eigenvalue $h(h-1)$, are also eigenvectors of the Euclidean kernel of the Bethe-Salpeter equation [19]. In this regime, the kernel of the Bethe-Salpeter equation is:

$$
K_{c}\left(\tau_{1}, \tau_{2} ; \tau_{3}, \tau_{4}\right) \propto \frac{\operatorname{sgn}\left(\tau_{13}\right) \operatorname{sgn}\left(\tau_{24}\right)}{\left|\tau_{13}\right|^{2 \Delta}\left|\tau_{24}\right|^{2 \Delta}\left|\tau_{34}\right|^{2-4 \Delta}}, \quad \Delta=1 / q,
$$

where the eigenvalues of $K_{c}$ depend on $q$ and $h$. Moreover, the allowed values of $h$ are constrained, because the Bethe-Salpeter equation for the OTOC selects the eigenvalue unity of $K_{c}$. For $q=4$, the leading contribution to the OTOC turns out to be $h=2$ and is given by [19]:

$$
\begin{aligned}
\frac{\mathcal{F}\left(\theta_{1} \ldots \theta_{4}\right)}{G\left(\theta_{12}\right) G\left(\theta_{34}\right)} & =\frac{6 \alpha_{0}}{\pi^{2} \alpha_{K}} \beta \mathcal{J} \sum_{|n| \geq 2} \frac{e^{i n\left(y^{\prime}-y\right)}}{n^{2}\left(n^{2}-1\right)}\left[\frac{\sin \frac{n x}{2}}{\tan \frac{x}{2}}-n \cos \frac{n x}{2}\right]\left[\frac{\sin \frac{n x^{\prime}}{2}}{\tan \frac{x^{\prime}}{2}}-n \cos \frac{n x^{\prime}}{2}\right] \\
x & =\theta_{12} \quad x^{\prime}=\theta_{34} \quad y=\frac{\theta_{1}+\theta_{2}}{2} \quad y^{\prime}=\frac{\theta_{3}+\theta_{4}}{2},
\end{aligned}
$$

where $\theta$ is the rescaled Euclidean time $\theta=\tau / \beta$. This equation must now be analytically continued to real time by choosing the operator insertions. We consider the contour shown in figure 8, which allows us to consider both the $\sigma$ - and $\alpha$-families simultaneously.

\footnotetext{
${ }^{6}$ We thank Subir Sachdev and Yingfei Gu for emphasizing that this should be the case.
} 


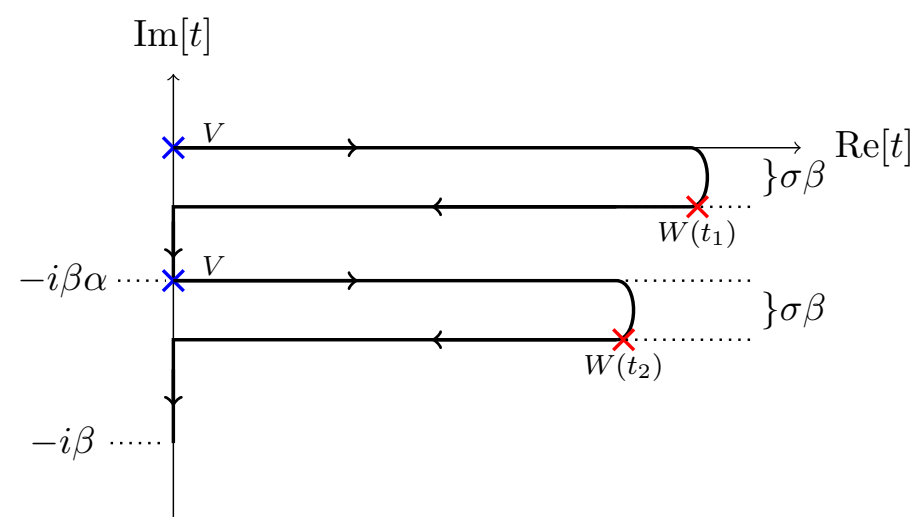

Figure 8. Extended Schwinger-Keldysh contour corresponding to the two-parameter OTOC $\operatorname{Tr}\left[\rho^{\alpha} W\left(t_{1}+i \sigma \beta\right) V \rho^{1-\alpha} W\left(t_{2}+i \sigma \beta\right) V\right]$.

More specifically, we choose

$$
\theta_{1}=i \frac{2 \pi t}{\beta}+2 \pi(\sigma+\alpha), \quad \theta_{2}=i \frac{2 \pi t}{\beta}+2 \pi \sigma, \quad \theta_{3}=2 \pi \alpha, \quad \theta_{4}=0 .
$$

In terms of $x, x^{\prime}, y, y^{\prime}$, we have:

$$
x=x^{\prime}=2 \pi \alpha, \quad y=i \frac{2 \pi t}{\beta}+2 \pi \sigma+\pi \alpha, \quad y^{\prime}=\pi \alpha .
$$

In order compute eq. (4.9) explicitly, we set $x=x^{\prime}$, sum over $n$ and then substitute eq. (4.10) to get:

$$
\begin{aligned}
\frac{\mathcal{F}(t)}{G(2 \pi \alpha) G(2 \pi \alpha)} \propto & \frac{1}{2}-\frac{\pi}{4}\left\{2 \pi \cot ^{2}(\pi \alpha)\left[(\alpha-1) \alpha+\sigma+i \frac{t}{\beta}\right]+(4 \alpha-2) \cot (\pi \alpha)\right. \\
& \left.+i \csc ^{2}(\pi \alpha) \sinh \left(\frac{2 \pi t}{\beta}-2 i \pi \sigma\right)\right\}
\end{aligned}
$$

which for large $t$ behaves as:

$$
\frac{\mathcal{F}(t)}{G(2 \pi \alpha) G(2 \pi \alpha)} \propto-i \frac{\pi^{2}}{4} \csc ^{2}(\pi \alpha) e^{-2 i \pi \sigma} e^{\frac{2 \pi t}{\beta}} .
$$

We first note that $\mathcal{F}(t)$ is symmetric over $\alpha \rightarrow 1-\alpha$, as expected. Second, the long-time regime is controlled by a growth rate given by $2 \pi / \beta$, independent of the distance between the forward branches $\alpha$. This confirms the contour dependence of the OTOC while the Lyapunov spectrum is nevertheless independent. The same contour dependence appears in 2-dimensional CFTs in the large central charge limit, where the OTOC is dominated by the Virasoro identity block [21]. In this case the contour-dependence always enters as prefactors of the $e^{\frac{2 \pi t}{\beta}}$ as in eq. (4.11).

\subsection{Study of the OTOC in SYK in the limit of large interaction order}

In the SYK model, analytical control is also possible when one increases the order of the interaction in the Hamiltonian (4.1), which is set by $q$ [19]. Here we consider the calculation 
of the Lyapunov exponent in SYK in the large- $q$ limit, and show that it is also contour independent.

We start with the two-point function in Euclidean signature in the large- $q$ expansion [19]:

$$
G(\tau) \underset{q \gg 1}{=} \frac{1}{2} \operatorname{sgn}(\tau)\left(1+\frac{1}{q} g(\tau)+O\left(q^{-2}\right)\right)
$$

where $g(\tau)$ is obtained by inserting the above ansatz in the saddle point equation for the two-point function. This gives the equation

$$
\partial_{\theta}^{2} g=2(\beta \mathcal{J})^{2} e^{g(\theta)}
$$

where $\theta=\tau / \beta \in[0,1)$ and $\mathcal{J}^{2}=q 2^{1-q} J^{2}$, and with boundary conditions $g(0)=g(1)=0$. The solution of eq. (4.13) is

$$
e^{g_{(0)}(\theta)}=\left[\frac{\cos \frac{\pi \nu}{2}}{\cos \left[\pi \nu\left(\frac{1}{2}-\theta\right)\right]}\right]^{2}, \quad \beta \mathcal{J}=\frac{\pi \nu}{\cos \frac{\pi \nu}{2}},
$$

with $\nu \in[0,1]$ parametrising the flow from weak $\beta J \sim 0$ coupling $(\nu \sim 0)$, to strong coupling $\beta J \gg 1(\nu \sim 1)$. The analytic continuation to real time reads

$$
\begin{aligned}
G^{R}(t) & =\theta(t)[G(\tau \rightarrow i t+\epsilon)-G(\tau \rightarrow i t-\epsilon)]=\theta(t)+\mathcal{O}(1 / q), \\
G^{(\alpha)}(t) & =G(\tau \rightarrow i t+\alpha \beta),
\end{aligned}
$$

for $\alpha=1 / 2$, and $G^{(\alpha)}(t)$ gives the Wightman function with operators separated by $i \beta / 2$. Instead of working in frequency space with eq. (4.8), we work in the time domain and use the following simplification for large $q$ :

$$
J^{2}(q-1) G(\tau)^{q-2} \underset{q \gg 1}{\widetilde{\sim}} J^{2} q 2^{2-q} \operatorname{sgn}(\tau)^{q-2} e^{g(\tau)}=2 \mathcal{J}^{2} e^{g(\tau)} .
$$

Therefore, using eqs. (4.15) and (4.16), the kernel of the Bethe-Salpeter equation, eq. (4.3), for large- $q$ is

$$
\begin{aligned}
K^{(\alpha)}\left(t_{1}, \ldots, t_{4}\right) & =J^{2}(q-1) G^{R}\left(t_{13}\right) G^{R}\left(t_{24}\right) G^{\alpha}\left(t_{34}\right)^{q-2} \\
& \underset{q \gg 1}{\simeq} \theta\left(t_{13}\right) \theta\left(t_{24}\right) 2 \mathcal{J}^{2} e^{g\left(\tau \rightarrow i t_{34}+\beta \alpha\right)} \\
& =\theta\left(t_{13}\right) \theta\left(t_{24}\right) \frac{2 \pi^{2} \nu^{2}}{\beta^{2} \cosh ^{2}\left(\frac{\pi \nu}{\beta}\left(t_{34}+i \eta\right)\right)}, \quad \eta=\beta(\alpha-1 / 2),
\end{aligned}
$$

where $0<\nu<1$ and we take $0<\alpha<1 / 2$. Finally, we use $\partial_{t} \theta(t)=\delta(t)$ to simplify the Bethe-Salpeter equation

$$
\begin{aligned}
\partial_{t_{1}} \partial_{t_{2}}\left[F\left(t_{1}, t_{2}\right)\right. & \left.=\int \mathrm{d} t_{3} \mathrm{~d} t_{4} K^{(\alpha)}\left(t_{1}, \ldots, t_{4}\right) F\left(t_{3}, t_{4}\right)\right] \\
\partial_{t_{1}} \partial_{t_{2}} F\left(t_{1}, t_{2}\right) & =2 \mathcal{J}^{2} e^{g\left(\tau \rightarrow i t_{12}+\beta \alpha\right)} F\left(t_{1}, t_{2}\right) .
\end{aligned}
$$


Making the ansatz $F\left(t, t^{\prime}\right)=e^{\lambda_{L} \frac{t+t^{\prime}}{2}} f\left(t-t^{\prime}\right)$ :

$$
\begin{gathered}
{\left[\partial_{t_{12}}^{2}+2 \mathcal{J}^{2} e^{g\left(\tau \rightarrow i t_{12}+\beta \alpha\right)}\right] f\left(t_{12}\right)=\frac{\lambda_{L}^{2}}{4} f\left(t_{12}\right),} \\
{\left[\partial_{t_{12}}^{2}+\frac{\pi^{2} \nu^{2}}{\beta^{2}} \frac{2}{\cosh ^{2}\left(\frac{\pi \nu}{\beta}\left(t_{12}+i \eta\right)\right)}\right] f\left(t_{12}\right)=\frac{\lambda_{L}^{2}}{4} f\left(t_{12}\right),} \\
{\left[-\partial_{y}^{2}-\frac{2}{\cosh ^{2}\left(y+i \frac{\pi \nu}{\beta} \eta\right)}\right] f(y)=-\left(\frac{\lambda_{L} \beta}{2 \pi \nu}\right)^{2} f(y) .}
\end{gathered}
$$

This is the Schrödinger equation with a complex Pöschl-Teller potential, which has a boundstate, $f(y)=\sqrt{\frac{\tan (\pi \nu \eta / \beta)}{4 \eta \cos (\pi \nu \eta / \beta)}} \operatorname{sech}\left(y+i \frac{\pi \nu}{\beta} \eta\right)$, with real eigenvalue $E=-1$. The value of the eigenenergy gives the value of the Lyapunov exponent $\lambda_{L}=2 \pi \nu / \beta$, which is independent on the contour parameter $\alpha$.

The large $q$ analysis allows us a qualitative insight into the role of the gapless nature of SYK by taking a closer look to the SYK-BSE eq. (4.8). Take the kernel of the BetheSalpeter equation in the regime where conformal symmetry is only weakly broken $\beta J \gg 1$. In this regime, the symmetric $\eta=0$ Wightman function is $G^{W}(t)=b\left[\frac{\pi}{\beta \cosh \frac{\pi t}{\beta}}\right]^{2 / q}, b^{q}=$ $\left(\frac{1}{2}-\frac{1}{q}\right) \tan (\pi / q) /\left(J^{2} \pi\right)[19]$. Consequently, the $\eta=0$ kernel $g_{l r}(\omega)$ is

$g_{l r}\left(\omega^{\prime}-\omega\right)=\int \mathrm{d} t e^{i \omega t} G^{W}(t)^{q-2}=b^{q-2}\left(\frac{\pi}{\beta}\right)^{2-4 / q} \frac{2^{1-4 / q}}{\Gamma\left(2-\frac{4}{q}\right)}\left|\Gamma\left(1-\frac{2}{q}-i \frac{\beta\left(\omega^{\prime}-\omega\right)}{2 \pi}\right)\right|^{2}$.

Using the identity $|\Gamma(a+i b)|^{2}=\Gamma(a)^{2} \prod_{k=0}^{\infty} \frac{1}{1+b^{2} /(a+k)^{2}}$, one immediately sees that this kernel is strongly peaked around the origin $\beta\left(\omega^{\prime}-\omega\right) \rightarrow 0$. On the other hand, changing the regularization changes the kernel by an overall factor $e^{(\alpha-1 / 2) \beta\left(\omega^{\prime}-\omega\right)}$. Thus, as the integral in eq. (4.8) is dominated by $\omega^{\prime} \sim \omega$, the dependence on the contour proportional to $e^{\eta\left(\omega^{\prime}-\omega\right)} \sim 1$ essentially drops out.

In a theory with gapped excitation, on the other hand, one can see for the case of the matrix model by numerically inspecting the expression of the on-shell kernel (A.3) and (A.4), that the kernels are peaked around the value of the gap. This gives a contribution of the order $e^{\eta \Delta_{\text {gap }}}$. Physically it may be seen as a consequence of a combination of an on-shell particle and anti-particle process that dominates the kernel.

\section{The Lyapunov spectrum and the Loschmidt echo}

In previous sections we have seen how the regularization dependence can afflict the commutator-squared. This shows that without more detailed specification one cannot directly relate this quantity to an observable that can be measured in experiments. We have also shown that the regularization dependence is dominant in the IR rather than the UV. This is analogous to Schwinger-Keldysh theory where contour dependence is related to the 
temperature, and the latter is a well known IR regulator. IR regularization issues are usually not solved by counterterms and renormalization. Instead they often encode physics on their own. This suggests that a way to resolve the regulator dependence is to define which member of family of "regularized" correlation functions computes a proper physical observable. The weakly coupled QFT result, through the mapping of the commutator-squared to a kinetic equation, indicates that the symmetrically regularized commutator-squared is the correct one.

Fortuitously this is the one almost exclusively studied in the literature and the one for which the MSS bound on chaos is derived. Nevertheless, one would like to understand from first principles why the symmetrized contour is an appropriate physical observable. The first attempt construction in the introduction points to the thermally averaged commutator-squared instead. In this section we show that the symmetrized commutatorsquared follows directly from an alternative measure of chaos, which is related to standard measurements of information spreading: the Loschmidt echo. This quantity contains not only the commutator-squared but also higher-order out-of time correlation functions. The Loschmidt echo and related quantities have been used in the context of quantum chaos for a long time [22-26]. Therefore, it is not surprising that the OTOCs may be extracted from echo spectroscopy as proposed in [27-30] and measured experimentally in [10]. More recently, the connection between the OTOC and the Loschmidt echo at infinite temperature was also explored in [31].

\subsection{Loschmidt echo}

The Loschmidt echo is based on a old thought experiment trying to disprove the irreversibility inherent in Boltzmann's equations by imaging a dynamical system where at time $t$ after $t_{0}=0$ one reverses all velocities and compare the resulting state at time $2 t$ with the original state. Microscopically the answer is of course identical, but supposing one makes a tiny "erroneous" perturbation at the time when one reverses all velocities, one immediately sees that in a chaotic non-integrable system the resulting state will be exponentially different from the original state.

This thought experiment can be directly mapped to a quantum quench experiment. One evolves a quantum state forward in time for a time $t$, perturbs it with an instantaneous quench $e^{i \delta W}$, evolves backward for the same time $t$ and projects onto the original state,

$$
M(t) \equiv\left\langle\psi\left|e^{i H t} e^{i \delta W} e^{-i H t}\right| \psi\right\rangle
$$

For a generic initial state, the echo will have a universal late time exponential fall off independent of the type of quench $W$ that encodes the lack of overlap between the initial and final state,

$$
M(t)=\left\langle\psi_{\text {initial }} \mid \psi_{\text {Loschmidt }}(2 t)\right\rangle \sim e^{-\lambda t}
$$

The Lyapunov exponent $\lambda$ is then a property of the system characterized by its Hamiltonian $H$ alone. 
The Loschmidt echo is the expectation value of a complex operator. To avoid phases one often takes the absolute value squared, which is known as the fidelity [32]

$$
F(t) \equiv\left|\left\langle\psi\left|e^{i H t} e^{i \delta W} e^{-i H t}\right| \psi\right\rangle\right|^{2} \sim\left|\frac{1}{1+\delta e^{\lambda t}}\right|^{2} \stackrel{t \rightarrow \infty}{\sim} e^{-2 \lambda t}
$$

The intermediate step is a well-known result from Jalabert and Pastawski [23]. A second practical step with an eye on experiment is to consider the fidelity for an ensemble of states, rather than a single state. Choosing the thermal ensemble one has

$$
\begin{aligned}
F(t) & \equiv\left|\frac{1}{Z_{\beta}} \sum_{\psi} e^{-\beta E[\psi]}\left\langle\psi\left|e^{i H t} e^{-i \delta W} e^{-i H t}\right| \psi\right\rangle\right|^{2} \\
& =\left|\operatorname{Tr} \rho e^{i H t} e^{-i \delta W} e^{-i H t}\right|^{2} \\
& =\operatorname{Tr} \rho e^{i H t} e^{-i \delta W} e^{-i H t} \rho e^{i H t} e^{i \delta W} e^{-i H t}+\mathcal{O}(1 / t) \\
& =\operatorname{Tr} \rho e^{-i \delta W(t)} \rho e^{i \delta W(t)} .
\end{aligned}
$$

Defining $e^{-i H t} \rho e^{i H t} \equiv X$ and $e^{-i \delta W} e^{-i H t} \rho e^{i H t} e^{i \delta W}=Y$, the fidelity $F(t)$ above is a specific case of the more general operator fidelity $\mathcal{F}=\operatorname{Tr} X^{\dagger} Y$ applied to density matrices as operators. ${ }^{7}$ Three remarks are in order. (1) In the intermediate step we used that the leading Lyapunov decay rate in $t$ is the same when computed via $\left|\operatorname{Tr} \rho e^{-i \delta W(t)}\right|^{2}$ or $\operatorname{Tr} \rho e^{-i \delta W(t)} \rho e^{i \delta W(t)}$. (2) Naively, as the late time Lyapunov exponent of interest is a property of the system and not of the initial state, the averaging should not matter. However, it is well known from classical dynamical systems that the late time behavior of an ensemble of classical trajectories is governed by Policott-Ruelle decay, rather than the microscopic exponential growth. Even though these are qualitatively related in weakly coupled theories, they are not quantitatively the same [11]. (3) Note both the symmetrized appearance of the density matrix, and the fact that the cumulative power of the density matrix is 2 . Computed through a path-integral this implies that the periodicity in Euclidean time is twice the inverse temperature $\beta=2 / T_{\text {phys }}$.

To connect with the commutator-squared, we expand to second order in $\delta$

$$
\begin{aligned}
F(t) & =\operatorname{Tr} \rho^{2}+\operatorname{Tr} \rho\left(-\delta^{2} W(t)^{2}\right) \rho+\operatorname{Tr} \rho(\delta W(t)) \rho \delta W(t) \\
& =\operatorname{Tr} \rho^{2}+\frac{\delta^{2}}{2} \operatorname{Tr}[\rho, W(t)][\rho, W(t)]+\ldots
\end{aligned}
$$

with the difference that the density matrix itself takes the role of the operator $V(0)$. The second time dependent term, the density-matrix commutator-squared, is a variant of the

\footnotetext{
${ }^{7}$ The operator fidelity is a weaker version of state fidelity encoding the notion of how close a state is to a maximally entangled one [33] or, if referring to teleportation, it quantifies the quality of the teleportation that can be achieved with the given state [34]. The state fidelity between two quantum states given by the density matrices $\rho_{0}$ and $\rho_{1}$ equals $[35,36]$ :
}

$$
F\left(\rho_{0}, \rho_{1}\right) \equiv \operatorname{Tr} \sqrt{\rho_{1}^{1 / 2} \rho_{0} \rho_{1}^{1 / 2}} .
$$


Wigner-Yanase-Dyson skew information.

$$
I_{\alpha}(\rho, A) \equiv \frac{1}{2} \operatorname{Tr}\left[i\left[\rho^{2 \alpha}, A^{\dagger}\right]\left(i\left[\rho^{2-2 \alpha}, A\right]\right)\right], 0 \leq \alpha \leq 1,
$$

for the symmetric value $\alpha=1 / 2$ [37]. Writing out the symmetric case for hermitian A,

$$
I_{\frac{1}{2}}(\rho, A)=(\operatorname{Tr} \rho A \rho A-\operatorname{Tr} \rho A A \rho)
$$

and replacing the thermal density matrix $\rho$ with a pure state density matrix,

$$
I_{\frac{1}{2}}(|\psi\rangle\langle\psi|, A)=-\left(\left\langle A^{2}\right\rangle-\langle A\rangle^{2}\right),
$$

one can recognize that the WYD skew information is an extension of the variance for pure states to mixed states. If, by the same argument as above, one may assume that it is dominated by some largest eigenvalue $\operatorname{Tr} \rho A \rho A \sim(\operatorname{Tr} \rho A)^{2}$, it computes something akin to the (largest eigenvalue) variance for the operator $\mathcal{O}=\rho A$. In that sense it is again natural that the density matrix appears with cumulative power 2. Put differently, in computing the WYD skew information the periodicity in Euclidean time is twice the inverse temperature $\beta=2 / T_{\text {phys }}$.

However, this is not yet the commutator-squared we are interested in. A guess might be the case where the thermal density matrix is rotated by a small similarity transformation $\rho=e^{i V} \rho_{0} e^{-i V}$. This is equivalent to an instantaneous quench by $V$ at time $t=1$. Then in the limit of small $\delta$ the late time fidelity equals

$$
\begin{aligned}
F(t) & =\operatorname{Tr} \rho e^{i H t} e^{-i \delta W} e^{-i H t} \rho e^{i H t} e^{i \delta W} e^{-i H t} \\
& =\operatorname{Tr} \rho_{0} e^{i V(0)} e^{-i \delta W(t)} e^{-i V(0)} \rho_{0} e^{i V(0)} e^{i \delta W(t)} e^{-i V(0)} \\
& =\operatorname{Tr} \rho_{0}^{2}+\frac{\delta^{2}}{2} \operatorname{Tr}\left[\rho_{0}, e^{i V(0)} W(t) e^{-i V(0)}\right]\left[\rho_{0}, e^{i V(0)} W(t) e^{-i V(0)}\right]+\ldots \\
& =\operatorname{Tr} \rho_{0}^{2}+\delta^{2}\left(\operatorname{Tr} \rho_{0} e^{i V(0)} W(t) e^{-i V(0)} \rho_{0} e^{i V(0)} W(t) e^{-i V(0)}-\operatorname{Tr} \rho_{0} e^{i V(0)} W(t)^{2} e^{-i V(0)} \rho_{0}\right) .
\end{aligned}
$$

The first and the last term can never give an OTOC; ignoring those, one has in the limit of small $V$

$$
\begin{aligned}
F(t)= & \operatorname{TOC}+\delta^{2}\left(\operatorname{Tr} \rho_{0} W(t) \rho_{0} W(t)+2 \operatorname{Tr} \rho_{0} W(t) \rho_{0}[V, W(t)]+\right. \\
& \left.+\operatorname{Tr} \rho_{0} W(t) \rho_{0}[V,[V, W(t)]]+\operatorname{Tr} \rho_{0}[V, W(t)] \rho_{0}[V, W(t)]+\ldots\right) .
\end{aligned}
$$

The two terms of order $\delta^{2}$ in the first line are also TOC. The terms on the second line contain the symmetric commutator-squared and a second term which is also an OTOC but on a different contour. ${ }^{8}$ As we know by now, generically the Lyapunov behavior of this other OTOC will be different. This is not yet the answer.

\footnotetext{
${ }^{8}$ Note that, at higher orders in $\delta$, the fidelity contains higher-order correlation functions, which are still represented by a Schwinger-Keldysh contour with only two folds but with multiple insertions of operators. These correlation functions differ from higher-point OTOCs in Schwinger-Keldysh contours with more than two folds [38]. The latter correspond to multiple repetitions of the Loschmidt experiment and, consequently, the largest growth rate is simply a multiple of the Lyapunov exponent of the 4-point function OTOC corresponding to a single repetition of the Loschmidt experiment.
} 
Tracing the origin of eq. (5.11), it is easy to see how the fidelity and the symmetrized commutator-squared are related. Eq. (5.11) follows from taking the long time limit and then taking $V$ and $\delta W$ small in the fundamental definition of the ensemble averaged fidelity - the first line of eq. (5.4). If, however, we take the limit of $V$ and $\delta W$ small, with $\rho=e^{i V} \rho_{0} e^{-i V}$, the ensemble averaged fidelity equals

$$
\begin{aligned}
F(t)= & \left|\operatorname{Tr} e^{i V(0)} \rho_{0} e^{-i V(0)} e^{-i \delta W(t)}\right|^{2} \\
= & \left|\operatorname{Tr} \rho_{0}\left(1-i \delta W(t)-\frac{\delta^{2}}{2} W(t)^{2}-\delta[V, W(t)]+\ldots\right)\right|^{2} \\
= & \left|\operatorname{Tr} \rho_{0}\right|^{2}-\delta \operatorname{Tr} \rho_{0} \operatorname{Tr} \rho_{0}[V, W(t)]-\delta \operatorname{Tr} \overline{\rho_{0}[V, W(t)]} \operatorname{Tr} \rho_{0} \\
& +\delta^{2} \operatorname{Tr} \overline{\rho_{0}[V, W(t)]} \operatorname{Tr} \rho_{0}[V, W(t)] .
\end{aligned}
$$

We now use the late time approximation, where we assume that $\rho_{0}[V, W(t)]$ is dominated by an eigenvalue $\operatorname{Eig}\left(\rho_{0}[V, W(t)]\right) \sim e^{\frac{1}{2}(\lambda+i \phi) t}$. In that limit, the middle two terms give a strongly oscillatory contribution, which is hard to measure. We therefore ignore it. As to the last term in eq. (5.12), there the late time limit allows us to make again the approximation

$$
\begin{aligned}
F(t) & =\ldots+\operatorname{Tr} \overline{\rho_{0}[V, W(t)]} \operatorname{Tr} \rho_{0}[V, W(t)] \\
& =\ldots+\operatorname{Tr} \overline{\rho_{0}[V, W(t)]} \rho_{0}[V, W(t)]+\mathcal{O}(1 / t) .
\end{aligned}
$$

We recognize precisely the symmetrized commutator-squared with one already noted difference. The cumulative power of the density matrix is 2 . This implies that the connection between the periodicity in Euclidean time and the physical temperature differs with a factor two compared to what the naive smearing procedured assumes: $\beta=2 / T_{\text {phys }}$. In particular this means the proper MSS bound on chaos should read $\lambda \leq \pi T_{\text {phys }}$.

The above is a strong argument that the natural observable which measures the symmetrized commutator-squared is the Loschmidt echo in the limit of small quenches first and late time subsequent with the sublety that $\beta=2 / T_{\text {phys }}$.

\section{Conclusion}

In this article we have explored the role of the regularization scheme of the commutatorsquared and of the OTOC. Quantum chaotic systems may display an exponential growth parametrized by a quantum Lyapunov exponent which is bounded by above $\lambda \leq 2 \pi k_{B} T / \hbar$ [3]. The proof of this bound involves regularising the OTOC by thermally spreading the operators. Purportedly, this is done to regulate short distance singularities and any physical property of a system should be independent of the short distance regularization scheme.

Here, we have shown that for those regularizations consisting on a contour with a $i \beta / 2$ separation between the forward branches, shifting the backwards branches induces a change in the decoherence factor, defined as the prefactor of the sum of the OTOCs [14]. Therefore, the decoherence factor cannot be a physical quantity as previously suggested. 
On the other hand, the Lyapunov exponent is the same for all of these contours, suggesting that indeed it may be measurable.

However, we have then shown that for a different choice of contours, where the separation between the forward branches is changed, the Lyapunov spectrum also depends on the contour chosen. While the contour dependence of the commutator-squared has been mostly overlooked in the literature, it is not surprising that this is the case. Similarly to the Wightman function, the commutator-squared involves operators inserted on forward and backward branches of the Schwinger-Keldysh contour, and so there is no reason to expect that it should be a physical quantity. Therefore, it is important to know how to extract physical information from it, in the same way that the spectral density, a physical quantity, may be obtained from the Wightman function, even though the Wightman function itself is not physical. The one notable exception in the literature is [5]. There, the authors studied many body chaos in a weakly interacting $2 \mathrm{D}$ system of fermions with quenched disorder and computed the Lyapunov exponent both for the unregularized $\eta=1 / 2$ case and the symmetrically regularized one, $\eta=0$ (in our notation). They indeed found that the two results disagree, and pointed out the regulator dependence of the OTOC. The conclusion that they drew is that, in the model considered, the only special feature of the symmetrically regularized OTOC is a particular cancellations of divergencies in the computation, but the physical meaning behind this correlator remained obscure.

Here we have performed a more thorough analysis showing the regulator dependence of the OTOC for two paradigmatic models, a weakly coupled $\phi^{4}$ matrix boson (at any $N$ ) and the SYK model. By comparing to ordinary Schwinger-Keldysh theory, we provide a simple diagrammatic proof regarding the reason why the choice of the contour affects the OTOC, although the Lyapunov spectrum becomes contour independent for theories that stay massless/gapless even at finite temperature. This is particularly relevant for the SYK model, which has been extensively studied over the last years. Its largest Lyaponuv exponent, which saturates the MSS bound, is indeed contour independent. Similarly, in 2-dimensional CFTs in the limit of large central charge where the vacuum dominance emerges, the Lyapunov exponent is insensitive to the choice of contour.

These detailed studies allow us to recognize that the regulator dependence is an IR issue, and not an alleviation of purported UV singularities. This means one has to take more care in understanding the role of the regulator as it may contain physical information. One crucial insight of our paper is to recognise the special physical meaning of the symmetrically regularised OTOC, by means of kinetic theory [11]. The OTOC computed on this contour is the one which one can properly claim to compute chaos or scrambling. That the fact that the bound on chaos holds for this physically meaningful definition of OTOC is remarkable and open new directions on possible still unknown dynamical constraints that the bound can impose.

This does then raise the question which simple observable naturally gives rise to such a symmetric insertion of a thermal density matrix. We proposed a simple observable, related to the operator fidelity, which contains information beyond the commutator-squared and can be measured experimentally using echo spectroscopy. The corollary of using this observable to define the OTOC is that is based on a double insertion of density matrices, 
i.e. the periodicity in Euclidean time is twice the inverse temperature. From this point of view the bound on chaos should read $\lambda \leq \pi k_{B} T_{\text {phys }} / \hbar$.

Overall, our results pose the question on the usefulness of the commutator-squared to probe quantum chaos. The contour dependence of the commutator-squared and of the Lyapunov spectrum extracted from it casts doubts on whether the commutator square is physical and how physical information should be extracted from it. However, even though a natural way to define chaotic quantum system is that in which the OTOC displays an exponential growth, this growth regime actually clashes with the other notion of a quantum chaotic theory that it should display random matrix behaviour. In the SYK model, even though one has exponential growth at shorter times similar to classical weakly interacting chaos, spectral properties, such as the spectral form factor, are similar to that of random matrix theory for times of order of $N \log (N)$ and larger [39-41]. This suggests that the model becomes truly quantum chaotic after this time-scale. A gorgeous example of true quantum chaos embodied by random matrix behaviour has been observed on the kicked Ising spin- $1 / 2$ chain for much shorter timescales [42]. There is no exponential growth in the OTOC in this model, which challenges the notion of how quantum chaos and especially maximal chaos should be defined.

\section{Acknowledgments}

The authors have benefited from discussions with Nikolay Gnezdilov, Razvan Gurau, Alexander Krikun, Tomaz Prosen, Moshe Rozali, Philippe Sabella-Garnier, and wish to thank Yingfei Gu and Subir Sachdev for emphasizing the contour independence of the SYK Lyapunov spectrum and correcting an earlier version.

This research was supported in part by a VICI award of the Netherlands Organization for Scientific Research (NWO), by the Netherlands Organization for Scientific Research/Ministry of Science and Education (NWO/OCW), and by the Foundation for Research into Fundamental Matter (FOM).

\section{A Numerical calculation in matrix model}

In this appendix we outline the simplifications used to solve numerically the Bethe-Salpeter equation eq. (3.18). Following [8], we define

$$
P=|\boldsymbol{p}|, \quad K=|\boldsymbol{k}|, \quad y=|\boldsymbol{k}-\boldsymbol{p}|
$$

and express the momentum integral as follows

$$
\int d^{3} k=2 \pi \int_{0}^{\infty} K^{2} d K \int_{|K-P|}^{K+P} \frac{y d y}{K P} .
$$

Rewriting eq. (3.18) in the time domain and replacing the momentum integral, we arrive at the simplified version of the Bethe-Salpeter equation, which we solve numerically following 
the strategy described in [8]:

$$
\begin{aligned}
\lambda_{L} f(P)= & \int_{0}^{\infty} \mathrm{d} K\left[\left(\cosh \left(\eta E_{+}\right) f(K)-\frac{f(P)}{3} \frac{\sinh \left(\frac{\beta E_{P}}{2}\right)}{\sinh \left(\frac{\beta E_{K}}{2}\right)}\right) I_{+}(P, K)\right. \\
& \left.+\left(\cosh \left(\eta E_{-}\right) f(K)-\frac{f(P)}{3} \frac{\sinh \left(\frac{\beta E_{P}}{2}\right)}{\sinh \left(\frac{\beta E_{K}}{2}\right)}\right) I_{-}(P, K)\right],
\end{aligned}
$$

where

$$
\begin{aligned}
& I_{+}(P, K) \equiv \frac{K}{(2 \pi)^{2} P 4 E_{P} E_{K}} \int_{|K-P|}^{K+P} \mathrm{~d} y y R\left(E_{+}, y\right)=\frac{\frac{3 \tilde{g} K}{(2 \pi)^{3} \beta P E_{P} E_{K}}}{\sinh \left(\beta E_{+} / 2\right)} \int_{|K-P|}^{K+P} \mathrm{~d} y \log \frac{\sinh x_{+}^{+}}{\sinh x_{-}^{+}} \\
& I_{-}(P, K) \equiv \frac{K}{(2 \pi)^{2} P 4 E_{P} E_{K}} \int_{|K-P|}^{K+P} \mathrm{~d} y y R\left(E_{-}, y\right)=\frac{\frac{3 \tilde{g} K}{(2 \pi)^{3} \beta P E_{P} E_{K}}}{\sinh \left(\beta E_{-} / 2\right)} \int_{|K-P|}^{K+P} \mathrm{~d} y \log \frac{1-e^{-2 x_{+}^{-}}}{1-e^{2 x_{-}^{-}}} \\
& x_{ \pm}^{+}=\frac{\beta}{4}\left(E_{+} \pm y \sqrt{1+\frac{4 m^{2}}{y^{2}-E_{+}^{2}}}\right) \quad x_{ \pm}^{-}=\frac{\beta}{4}\left(E_{-} \pm y \sqrt{1+\frac{4 m^{2}}{y^{2}-E_{-}^{2}}}\right),
\end{aligned}
$$

and we defined $\tilde{g}=g^{4}\left(N^{2}+5\right) /(4 \cdot 144)$.

Open Access. This article is distributed under the terms of the Creative Commons Attribution License (CC-BY 4.0), which permits any use, distribution and reproduction in any medium, provided the original author(s) and source are credited.

\section{References}

[1] S.H. Shenker and D. Stanford, Black holes and the butterfly effect, JHEP 03 (2014) 067 [arXiv: 1306.0622] [INSPIRE].

[2] S.H. Shenker and D. Stanford, Multiple Shocks, JHEP 12 (2014) 046 [arXiv:1312.3296] [INSPIRE].

[3] J. Maldacena, S.H. Shenker and D. Stanford, A bound on chaos, JHEP 08 (2016) 106 [arXiv: 1503.01409] [INSPIRE].

[4] D. Chowdhury and B. Swingle, Onset of many-body chaos in the $O(N)$ model, Phys. Rev. D 96 (2017) 065005 [arXiv: 1703.02545] [INSPIRE].

[5] Y. Liao and V. Galitski, Nonlinear $\sigma$-model approach to many-body quantum chaos: Regularized and unregularized out-of-time-ordered correlators, Phys. Rev. B 98 (2018) 205124 [arXiv: 1807.09799] [INSPIRE].

[6] H. Matsumoto, Y. Nakano, H. Umezawa, F. Mancini and M. Marinaro, Thermo Field Dynamics in Interaction Representation, Prog. Theor. Phys. 70 (1983) 599 [InSPIRE].

[7] H. Matsumoto, Y. Nakano and H. Umezawa, An equivalence class of quantum field theories at finite temperature, J. Math. Phys. 25 (1984) 3076 [InSPIRE].

[8] D. Stanford, Many-body chaos at weak coupling, JHEP 10 (2016) 009 [arXiv:1512.07687] [INSPIRE]. 
[9] A. Das et al., Light-Cone Spreading of Perturbations and the Butterfly Effect in a Classical Spin Chain, Phys. Rev. Lett. 121 (2018) 024101 [arXiv:1711.07505].

[10] M. Gärttner, J.G. Bohnet, A. Safavi-Naini, M.L. Wall, J.J. Bollinger and A.M. Rey, Measuring out-of-time-order correlations and multiple quantum spectra in a trapped-ion quantum magnet, Nature Phys. 13 (2017) 781.

[11] S. Grozdanov, K. Schalm and V. Scopelliti, Kinetic theory for classical and quantum many-body chaos, Phys. Rev. E 99 (2019) 012206 [arXiv: 1804.09182] [INSPIRE].

[12] R. van Zon, H. van Beijeren and C. Dellago, Largest Lyapunov exponent for many particle systems at low densities, Phys. Rev. Lett. 80 (1998) 2035.

[13] H. van Beijeren, R. van Zon and J.R. Dorfman, Kinetic Theory Estimates for the Kolmogorov-Sinai Entropy and the Largest Lyapunov Exponents for Dilute, Hard-Ball Gases and for Dilute, Random Lorentz Gases, in Hard Ball Systems and the Lorentz Gas, D. Szász eds., Springer, Berlin Germany (2000) [chao-dyn/9909034].

[14] A. Kitaev and S.J. Suh, The soft mode in the Sachdev-Ye-Kitaev model and its gravity dual, JHEP 05 (2018) 183 [arXiv: 1711.08467] [INSPIRE].

[15] M. Blake, H. Lee and H. Liu, A quantum hydrodynamical description for scrambling and many-body chaos, JHEP 10 (2018) 127 [arXiv:1801.00010] [INSPIRE].

[16] Y. Gu and A. Kitaev, On the relation between the magnitude and exponent of OTOCs, JHEP 02 (2019) 075 [arXiv: 1812.00120] [INSPIRE].

[17] Y. Werman, S.A. Kivelson and E. Berg, Quantum chaos in an electron-phonon bad metal, arXiv:1705.07895 [INSPIRE].

[18] M.J. Klug, M.S. Scheurer and J. Schmalian, Hierarchy of information scrambling, thermalization and hydrodynamic flow in graphene, Phys. Rev. B 98 (2018) 045102 [arXiv: 1712.08813] [INSPIRE].

[19] J. Maldacena and D. Stanford, Remarks on the Sachdev-Ye-Kitaev model, Phys. Rev. D 94 (2016) 106002 [arXiv:1604.07818] [INSPIRE].

[20] A.M. García-García, B. Loureiro, A. Romero-Bermúdez and M. Tezuka, Chaotic-Integrable Transition in the Sachdev-Ye-Kitaev Model, Phys. Rev. Lett. 120 (2018) 241603 [arXiv: 1707.02197] [INSPIRE].

[21] D.A. Roberts and D. Stanford, Diagnosing Chaos Using Four-Point Functions in Two-Dimensional Conformal Field Theory, Phys. Rev. Lett. 115 (2015) 131603 [arXiv: 1412.5123] [INSPIRE].

[22] H.M. Pastawski, P.R. Levstein and G. Usaj, Quantum Dynamical Echoes in the Spin Diffusion in Mesoscopic Systems, Phys. Rev. Lett. 75 (1995) 4310.

[23] R.A. Jalabert and H.M. Pastawski, Environment-independent decoherence rate in classically chaotic systems, Phys. Rev. Lett. 86 (2001) 2490 [cond-mat/0010094].

[24] T. Prosen, General relation between quantum ergodicity and fidelity of quantum dynamics, Phys. Rev. E 65 (2002) 036208 [quant-ph/0106149v2].

[25] T. Prosen and M. Znidaric, Stability of quantum motion and correlation decay, J. Phys. A 35 (2002) 1455 [nlin/0111014v2].

[26] F. Haug, M. Bienert, W.P. Schleich, T.H. Seligman and M.G. Raizen, Motional stability of the quantum kicked rotor: A fidelity approach, Phys. Rev. A 71 (2005) 1. 
[27] G. Zhu, M. Hafezi and T. Grover, Measurement of many-body chaos using a quantum clock, Phys. Rev. A 94 (2016) 062329 [arXiv:1607.00079] [InSPIRE].

[28] N.Y. Yao et al., Interferometric Approach to Probing Fast Scrambling, arXiv:1607.01801 [INSPIRE].

[29] B. Swingle, G. Bentsen, M. Schleier-Smith and P. Hayden, Measuring the scrambling of quantum information, Phys. Rev. A 94 (2016) 040302 [arXiv:1602.06271] [INSPIRE].

[30] J. Kurchan, Quantum bound to chaos and the semiclassical limit, arXiv:1612.01278 [INSPIRE].

[31] B. Yan, L. Cincio and W.H. Zurek, Information Scrambling and Loschmidt Echo, arXiv: 1903.02651.

[32] T. Prosen, T.H. Seligman and M. Žnidarič, Theory of Quantum Loschmidt Echoes, Prog. Theor. Phys. Suppl. 150 (2003) 200 [quant-ph/0304104].

[33] C.H. Bennett, G. Brassard, C. Crepeau, R. Jozsa, A. Peres and W.K. Wootters, Teleporting an unknown quantum state via dual classical and Einstein-Podolsky-Rosen channels, Phys. Rev. Lett. 70 (1993) 1895 [INSPIRE].

[34] C.H. Bennett, D.P. DiVincenzo, J.A. Smolin and W.K. Wootters, Mixed state entanglement and quantum error correction, Phys. Rev. A 54 (1996) 3824 [quant-ph/9604024] [INSPIRE].

[35] A. Uhlmann, The "transition probability" in the state space of a $*$-algebra, Rept. Math. Phys. 9 (1976) 273 [INSPIRE].

[36] R. Jozsa, Fidelity for Mixed Quantum States, J. Mod. Opt. 41 (1994) 2315.

[37] E.P. Wigner and M.M. Yanase, Information contents of distributions, Proc. Nat. Acad. Sci. 49 (1963) 910.

[38] F.M. Haehl and M. Rozali, Effective Field Theory for Chaotic CFTs, JHEP 10 (2018) 118 [arXiv: 1808.02898] [INSPIRE].

[39] J.S. Cotler et al., Black Holes and Random Matrices, JHEP 05 (2017) 118 [Erratum ibid. 09 (2018) 002] [arXiv:1611.04650] [INSPIRE].

[40] A.M. García-García and J.J.M. Verbaarschot, Spectral and thermodynamic properties of the Sachdev-Ye-Kitaev model, Phys. Rev. D 94 (2016) 126010 [arXiv:1610.03816] [INSPIRE].

[41] A.M. García-García and J.J.M. Verbaarschot, Analytical Spectral Density of the Sachdev-Ye-Kitaev Model at finite N, Phys. Rev. D 96 (2017) 066012 [arXiv:1701.06593] [INSPIRE].

[42] B. Bertini, P. Kos and T. Prosen, Exact Spectral Form Factor in a Minimal Model of Many-Body Quantum Chaos, Phys. Rev. Lett. 121 (2018) 264101 [arXiv:1805.00931] [INSPIRE]. 